Article

\title{
Dietary Factors and Female Breast Cancer Risk: A Prospective Cohort Study
}

\author{
Ji Hyun Kim ${ }^{1}{ }^{(1)}$, Jeonghee Lee ${ }^{2}$, So-Youn Jung ${ }^{3}$ and Jeongseon Kim ${ }^{2, *}$ \\ 1 Department of Cancer Control and Population Health, Graduate School of Cancer Science and Policy, \\ National Cancer Center, 323 Ilsan-ro, Ilsandong-gu, Goyang-si 10408, Gyeonggi-do, Korea; \\ 1601009@ncc.re.kr \\ 2 Department of Cancer Biomedical Science, Graduate School of Cancer Science and Policy, \\ National Cancer Center, 323 Ilsan-ro, Ilsandong-gu, Goyang-si 10408, Gyeonggi-do, Korea; \\ jeonghee@ncc.re.kr \\ 3 Center for Breast Cancer, National Cancer Center Hospital, National Cancer Center, 323 Ilsan-ro, \\ Ilsandong-gu, Goyang-si 10408, Gyeonggi-do, Korea; goje1@ncc.re.kr \\ * Correspondence: jskim@ncc.re.kr; Tel.: +82-31-920-2570; Fax: +82-31-920-2579
}

Received: 20 September 2017; Accepted: 5 December 2017; Published: 7 December 2017

\begin{abstract}
Breast cancer is the leading cause of cancer in females and has become a major global health priority. This prospective cohort study investigated the association of dietary factors, including food items and dietary habits, with the risk of breast cancer in Korean women. Study participants were women aged 30 years or older, recruited from the National Cancer Center in South Korea between August 2002 and May 2007. They were followed until December 2014 using the Korea Central Cancer Registry to identify breast cancer cases. Among 5046 non-pre-diagnosed cancer participants, 72 breast cancer cases were prospectively identified. Participants with breast cancer had a significantly higher educational level (college or higher: $58.3 \%$ vs. $39.5 \%, p=0.01$ ), were more likely to have ever smoked $(22.2 \%$ vs. $7.8 \%$, $p<0.001)$, and were more likely to have a history of benign breast tumors ( $10 \%$ vs. $4 \%, p=0.02)$ than non-cases. Consumption of grilled meat conferred a significantly higher risk of breast cancer in all women (hazard ratio (HR) 1.77, 95\% confidence interval (CI) 1.09-2.85) and in postmenopausal women (HR 3.06, 95\% CI 1.31-7.15). High-cholesterol food intake was associated with a higher risk in all women (HR 1.69, 95\% CI 1.01-2.82). Irregular meal intake was associated with an elevated risk in all women (HR 2.19, 95\% CI 1.20-3.98, $p$ for trend = 0.01) and in premenopausal women (HR 2.35, 95\% CI 1.13-4.91, $p$ for trend $=0.03$ ). Our findings suggest that grilled meat and high-cholesterol food intake and irregular eating habits may be associated with a higher risk of breast cancer. Further studies with longer follow-up periods that include information on portion size, hormone receptor status, carcinogen levels in grilled meat, and a classification of foods by source are required.
\end{abstract}

Keywords: food groups; dietary habits; dietary factors; Korean; breast cancer; prospective cohort study

\section{Introduction}

Cancer is the leading cause of death worldwide and has become a major public health problem [1,2]. In Korea, cancer accounted for $28.3 \%$ of deaths in 2013 [3]. Of the various cancers, breast cancer is the most frequently diagnosed in women worldwide [1]. In Korea, the incidence of breast cancer has continually increased in the past 20 years [4] and was the second leading cause of female cancer in both incidence and prevalence in 2013. According to the Korean Central Cancer Registry (KCCR), the age-standardized incidence of breast cancer was 45.7 per 100,000 women, and the annual percent change between 1999 and 2013 was $5.9 \%$ in women [3].

Diet is considered a modifiable risk factor and accounts for approximately $35 \%$ of all cancer causes. Therefore, it is important to identify the dietary risk factors of cancer [5-8]. The westernization of 
Korea, including the rapid development and socioeconomic growth that have occurred since the 1950s, is speculated to have affected the incidence of cancer through drastic changes in lifestyle, including reproductive factors, physical activity, and diet [9-12]. Specifically, a rapid and unique transition in dietary patterns has emerged, as reflected by changes from the traditional diet based on grains and vegetables to a Western diet of predominantly meat and animal food products [13-15]. Vegetable intake may be inversely associated with cancer, but vegetables are generally consumed in pickled or salted forms in Korea, thereby increasing sodium intake, which is a dietary risk factor of cancer [16,17].

Previous epidemiological studies have addressed the dietary risk factors of breast cancer, considering nutrient or food items alone and together with dietary patterns [18-24]. Some studies found that higher intakes of alcohol [18], red and processed meat [19], and animal fat [20,21], and lower intakes of fruits and vegetables [22] and total dietary fiber [23], may be associated with a higher risk of breast cancer. Prudent/healthy dietary patterns reportedly decrease this risk, whereas no such evidence has been shown for Western/unhealthy diets [24]. However, the results have been inconsistent, and no clear association of breast cancer risk other than with alcohol consumption has been identified to date $[7,25,26]$.

Dietary factors tend to differ by population due to socioeconomic status, ethnicity, and culture [27]. Therefore, risk factors for cancer in Korean populations may differ from those in Western countries, where most studies have been conducted [25,28]. Moreover, most previous studies in Korea on dietary risk factors of breast cancer were restricted to case-control studies; therefore, prospective cohort studies are needed to determine the exact associations [29].

Accordingly, the aim of this prospective cohort study was to investigate the association of dietary factors with the risk of breast cancer among all women and in groups stratified by menopausal status: premenopausal and postmenopausal women.

\section{Materials and Methods}

\subsection{Study Population}

The source population included 14,531 men and women aged 30 years and older enrolled at the Center for Cancer Prevention and detection at the National Cancer Center in South Korea between August 2002 And May 2007. Male subjects were excluded, resulting in the selection of 6477 female subjects. A total of 1276 subjects were excluded for having an incomplete short-form Food Frequency Questionnaire (FFQ), and 155 subjects were excluded due to a diagnosis with any type of cancer before enrollment. Potential breast cancer cases (International Classification of Diseases-10 Code C50) were ascertained by linkage to the 2014 KCCR database, which provides information on cancer incidence by following case classifications in Korea. after baseline examination of general characteristics and dietary factors (16 food groups and six dietary habits), the participants were followed up and contributed to the person-years until whichever came first: the date of any cancer diagnosis except for non-melanoma skin cancer (ICD-10 Code C44), death, or the end of the follow-up (December 2014). Seventy-two subjects developed breast cancer, and 4974 subjects were identified as breast cancer non-cases (Figure 1). Written informed consent was obtained from all participants, and the study protocol was approved by the institutional review board of the National Cancer Center (No. NCCNCS-07-077). 


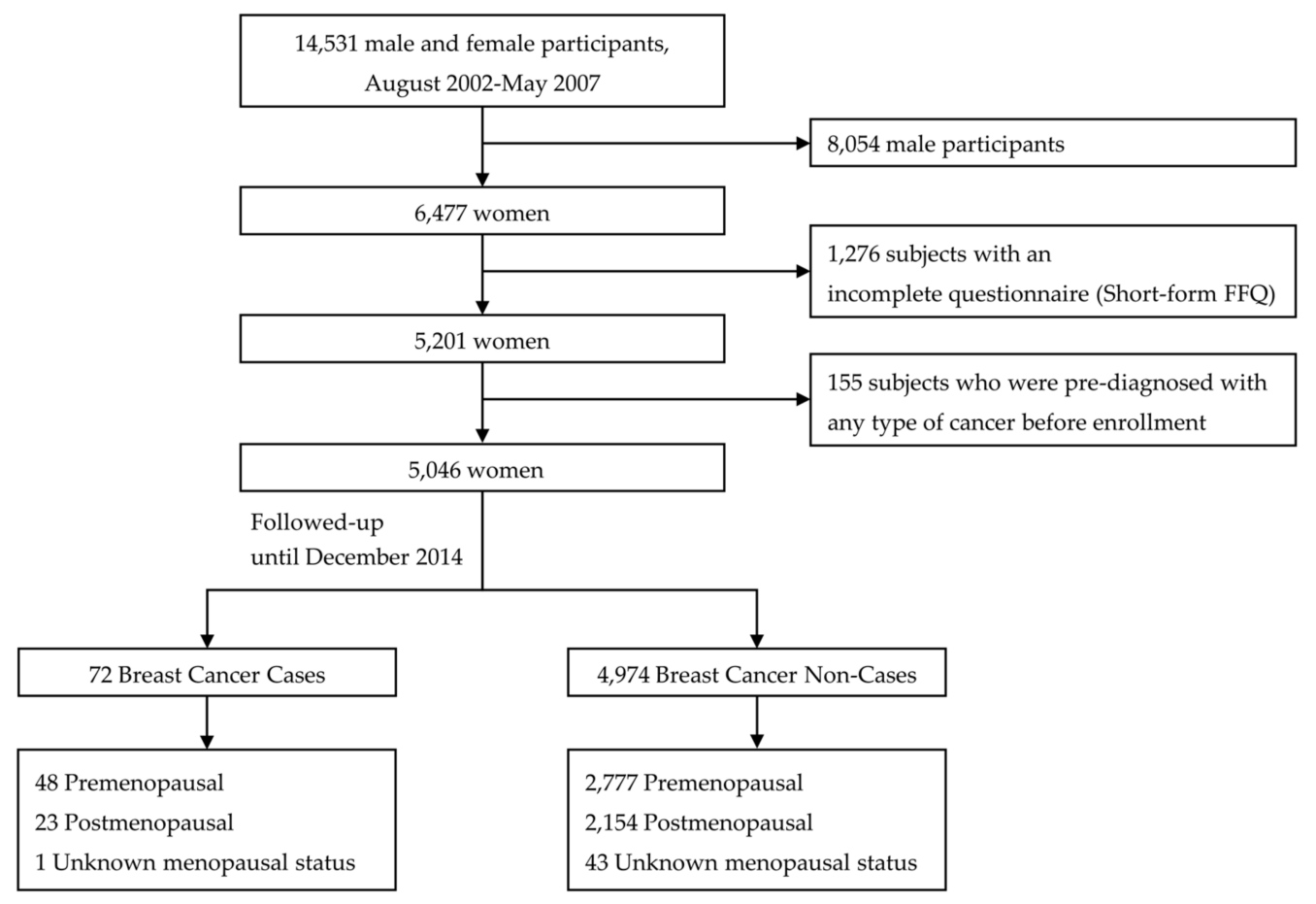

Figure 1. Flow chart of the study subjects.

\subsection{Data Collection and Management}

At the time of enrollment, all participants were asked to complete self-administered questionnaires regarding socio-demographic characteristics (e.g., age, education, occupation, household income, and marital status), family history of cancer, cigarette and alcohol consumption, regular exercise habits, history of benign breast tumor, and reproductive factors (e.g., age at menarche, menopausal status, age at menopause, type of menopause, postmenopausal hormone use, parity, delivery frequency, lactation, and oral contraceptive use). Height and weight were measured using an InBody 3.0 (Biospace, Seoul, Korea) body composition analyzer or the X-SCAN PLUS II Body Composition Analyzer (Jawon Medical, Gyeongsan, Korea), and body mass index (BMI) was calculated as weight $(\mathrm{kg}) / \mathrm{height}\left(\mathrm{m}^{2}\right)$.

The FFQ assessed 16 food groups: cereals, salty vegetables and seafood, light-colored vegetables, green-yellow vegetables, seaweed, fruit, grilled meat, healthy protein foods, dairy foods, bony fish, fried foods, high-cholesterol foods, animal fat-rich foods, sweet foods, fast foods, and caffeinated drinks (see Appendix A). The participants were asked to record the frequency of their current intake of each item according to eight categories: consumed rarely, once a month, two to three times a month, once a week, two to three times a week, four to six times a week, once a day, or more than two times a day. This FFQ has been used in several epidemiological studies on dietary factors related to chronic diseases [30-32] and health behaviors [33]. In our previous study, the FFQ was validated as a reference standard in 1401 participants from the source cohort based on a comparison with three-day dietary records. Cross-classification between the two methods of the distribution of subjects by tertiles indicated good agreement, ranging from $38 \%$ to $96 \%$ depending on the food group and nutrient [30].

Six questionnaires examining dietary habits were also assessed: meal frequency (three times a day, two times a day, irregular); breakfast frequency (always, often, not at all); meal time regularity (always regular, often regular, always irregular); meal speed (slow, average, fast); frequency of overeating (less than once a week, two to three times a week, more than four times a week); and the use of additional spices such as soy sauce, table salt, soybean paste, or hot pepper paste (often, sometimes, never). 


\subsection{Statistical Analysis}

The characteristics of the study subjects were compared using Student's $t$-test, chi-square test, or Fisher's exact test. As the distribution of intake frequencies of each food item was skewed, the FFQ items were reclassified into two categories, low and high consumption, based on the median distribution of non-cases. A Cox proportional hazards model with person-years as the underlying time metric was used to evaluate the hazard ratio (HR) and 95\% confidence interval (CI) of breast cancer for each dietary factor, including 16 food groups and six dietary habits. Multivariate Model 1 was adjusted for only significant factors of our study subjects' general characteristics (Table 1), and Model 2 was adjusted for potential confounders according to other breast cancer researches [34,35]. Model 1 of all women was adjusted for age, smoking status (current, ex, non-smoker), education status (elementary school or less, middle school, high school, college or higher), and benign breast tumor history (yes, no). Premenopausal women were adjusted for age and smoking status (current, ex, non-smoker), and postmenopausal women was adjusted for age, education status (elementary school or less, middle school, high school, college or higher), and benign breast tumor history (yes, no). Model 2 was adjusted for age, BMI (<23.0, 23.0 to $<25.0, \geq 25 \mathrm{~kg} / \mathrm{m}^{2}$ ), family history of breast cancer (yes, no), smoking status (current, ex, non-smoker), alcohol consumption (current, ex, non-drinker), physical activity (yes, no), age at menarche $(\leq 13,14,15, \geq 16$ years), parity $(0,1,2, \geq 3)$, oral contraceptive use (yes, no), and benign breast tumor history (yes, no) in premenopausal women. In all women, we additionally adjusted for hormone use and menopausal status (premenopausal, postmenopausal hormone users, postmenopausal hormone non-users, unknown menopausal status), and age at menopause (premenopausal, menopause at $<46,46-48,49-51$, $\geq 52$ years, unknown menopausal status). In postmenopausal women, we additionally adjusted for hormone use (yes, no) and age at menopause ( $<46,46-48,49-51, \geq 52$ years). All statistical analyses were performed using SAS software (version 9.4, SAS Institute, Cary, NC, USA), and the level of significance was set at $p<0.05$.

\section{Results}

A total of 5046 Korean women were eligible for this analysis. During a mean follow-up of 9.46 years, 72 women were diagnosed with breast cancer. The subjects with breast cancer had a significantly higher educational level in the all group (college or higher: $58.3 \%$ vs. $39.5 \%, p=0.01$ ) and the postmenopausal group (college or higher: $56.5 \%$ vs. $270 \%, p=0.04$ ), were more likely to be ever-smokers in the all $(22.2 \%$ vs. $7.8 \%, p<0.001)$ and premenopausal groups $(27.1 \%$ vs. $9.6 \%$, $p<0.001)$, and more frequently had a history of benign breast tumor in the all $(9.7 \%$ vs. $3.7 \%, p=0.02)$ and postmenopausal groups $(21.7 \%$ vs. $3.3 \%, p=0.001)$ than did non-cases (Table 1$)$.

Table 2 shows the intake frequencies of the 16 food groups based on the short-form FFQ and their association with breast cancer risk in all women. Consumption of grilled meat was associated with a significantly higher risk of breast cancer in the age-adjusted model, and this trend was maintained in multivariate Model 1 (HR 1.66, 95\% CI 1.03-2.68) and Model 2 (HR 1.77, 95\% CI 1.09-2.85). High-cholesterol food intake was positively associated with a higher risk only in the age-adjusted model (HR 1.71, 95\% CI 1.03-2.85) and multivariate Model 2 (HR 1.69, 95\% CI 1.01-2.82). Among the premenopausal women, there were no differences between the cases and non-cases (Table 3). In postmenopausal women, grilled meat consumption was associated with an elevated risk of breast cancer in both multivariate Model 1 (HR 2.41, 95\% CI 1.05-5.54) and Model 2 (HR 3.06, 95\% CI 1.31-7.15) (Table 4).

Table 5 shows the six dietary habits assessed and their associations with breast cancer risk in all women. Irregular meal intake was associated with an elevated risk of breast cancer in both multivariate Model 1 (HR 2.37, 95\% CI 1.31-4.27, $p$ for trend = 0.006) and Model 2 (HR 2.19, 95\% CI 1.20-3.98, $p$ for trend $=0.01$ ). A similar finding regarding irregular meal intake was observed in premenopausal women in both multivariate Model 1 (HR 2.15, 95\% CI 1.06-4.40, $p$ for trend $=0.044$ ) and Model 2 (HR 2.35, 95\% CI 1.13-4.91, $p$ for trend = 0.03) (Table 6), but not in postmenopausal women (Table 7). 
Table 1. General characteristics of the study subjects ${ }^{\text {a }}$.

\begin{tabular}{|c|c|c|c|c|c|c|c|c|c|}
\hline \multirow{2}{*}{ Characteristics } & \multicolumn{3}{|c|}{ Total $(n=5046)$} & \multicolumn{3}{|c|}{ Premenopausal $(n=2825)$} & \multicolumn{3}{|c|}{ Postmenopausal $(n=2177)$} \\
\hline & Non-Cases $(n=4974)$ & Cases $(n=72)$ & $p$ Value & Non-Cases $(n=2777)$ & Cases $(n=48)$ & $p$ Value & Non-Cases $(n=2154)$ & Cases $(n=23)$ & $p$ Value \\
\hline \multicolumn{10}{|l|}{ Age (years) } \\
\hline$<40$ & 1025 (20.6) & $10(13.9)$ & 0.06 & $1000(36.0)$ & $10(20.8)$ & 0.09 & $15(0.7)$ & $0(0.0)$ & 0.33 \\
\hline $40-49$ & $1951(39.2)$ & $39(54.2)$ & & 1599 (57.6) & $34(70.8)$ & & $336(15.6)$ & $5(21.7)$ & \\
\hline $50-59$ & $1390(28.0)$ & $14(19.4)$ & & $177(6.4)$ & $4(8.3)$ & & $1201(55.8)$ & $9(39.1)$ & \\
\hline $60+$ & $608(12.2)$ & $9(12.5)$ & & $1(0.0)$ & $0(0.0)$ & & $602(28.0)$ & $9(39.1)$ & \\
\hline \multicolumn{10}{|l|}{ BMI $\left(\mathrm{kg} / \mathrm{m}^{2}\right)$} \\
\hline$<23.0$ & $2692(54.1)$ & $41(56.9)$ & 0.89 & $1771(63.8)$ & $32(66.7)$ & 0.50 & $897(41.6)$ & $9(39.1)$ & 0.30 \\
\hline $23.0-<25.0$ & $1170(23.5)$ & $16(22.2)$ & & $547(19.7)$ & $11(22.9)$ & & $614(28.5)$ & $4(17.4)$ & \\
\hline$\geq 25.0$ & $1112(22.4)$ & $15(20.8)$ & & $459(16.5)$ & $5(10.4)$ & & $643(29.9)$ & $10(43.5)$ & \\
\hline Body fat (\%) & $29.21 \pm 5.35$ & $29.03 \pm 5.73$ & 0.78 & $27.88 \pm 5.17$ & $27.91 \pm 5.52$ & 0.99 & $30.88 \pm 5.09$ & $31.36 \pm 5.67$ & 0.66 \\
\hline \multicolumn{10}{|l|}{ Education level } \\
\hline Elementary school or less & $529(10.6)$ & $3(4.2)$ & 0.01 & $79(2.8)$ & $0(0.0)$ & 0.42 & $449(20.8)$ & $3(13.0)$ & 0.04 \\
\hline Middle school & $443(8.9)$ & $5(6.9)$ & & $158(5.7)$ & $3(6.3)$ & & $282(13.1)$ & $2(8.7)$ & \\
\hline High school & 1957 (39.3) & $21(29.2)$ & & $1146(41.3)$ & $16(33.3)$ & & $794(36.9)$ & $5(21.7)$ & \\
\hline College or higher & $1962(39.5)$ & $42(58.3)$ & & $1365(49.2)$ & $29(60.4)$ & & $581(27.0)$ & $13(56.5)$ & \\
\hline \multicolumn{10}{|l|}{ Occupation } \\
\hline Housewife & 3045 (61.2) & $40(55.6)$ & 0.33 & $1584(57.0)$ & $27(56.3)$ & 0.56 & $1443(67.0)$ & $13(56.5)$ & 0.37 \\
\hline Professional, office worker & $740(14.9)$ & $16(22.2)$ & & 537 (19.3) & $12(25.0)$ & & $192(8.9)$ & $4(17.4)$ & \\
\hline Sales, service & $592(11.9)$ & $7(9.7)$ & & $381(13.7)$ & $4(8.3)$ & & $208(9.7)$ & $3(13.0)$ & \\
\hline Agriculture, labor, unemployed, other & $463(9.3)$ & $8(11.1)$ & & $223(8.0)$ & $5(10.4)$ & & $236(11.0)$ & $3(13.0)$ & \\
\hline \multicolumn{10}{|l|}{ Household income $(10,000$ won/month) } \\
\hline$<200$ & $768(15.4)$ & $6(8.3)$ & 0.06 & $270(9.7)$ & $3(6.3)$ & 0.10 & $494(22.9)$ & $3(13.0)$ & 0.42 \\
\hline $200-<400$ & $1386(27.9)$ & $16(22.2)$ & & $807(29.1)$ & $8(16.7)$ & & $569(26.4)$ & $8(34.8)$ & \\
\hline$\geq 400$ & $2226(44.8)$ & $41(56.9)$ & & $1439(51.8)$ & $31(64.6)$ & & $766(35.6)$ & $10(43.5)$ & \\
\hline \multicolumn{10}{|l|}{ Marital status } \\
\hline Unmarried & $165(3.3)$ & $3(4.2)$ & 0.90 & $147(5.3)$ & $2(4.2)$ & 0.93 & $17(0.8)$ & $1(4.4)$ & 0.06 \\
\hline Married & $4378(88.0)$ & $62(86.1)$ & & $2499(90.0)$ & $45(93.8)$ & & $1847(85.8)$ & $17(73.9)$ & \\
\hline Divorced/widowed & $384(7.7)$ & $6(8.3)$ & & $112(4.0)$ & $1(2.1)$ & & $267(12.4)$ & $5(21.7)$ & \\
\hline Family history of breast cancer (Yes) & $168(3.4)$ & $4(5.6)$ & 0.31 & $91(3.3)$ & $2(4.2)$ & 0.67 & $76(3.5)$ & $2(8.7)$ & 0.21 \\
\hline \multicolumn{10}{|l|}{ Smoking status } \\
\hline Current smokers & $267(5.4)$ & $9(12.5)$ & $<0.001$ & $196(7.1)$ & 7 (14.6) & $<0.001$ & $68(3.2)$ & $2(8.7)$ & 0.21 \\
\hline Ex-smokers & $117(2.4)$ & $7(9.7)$ & & $70(2.5)$ & $6(12.5)$ & & $45(2.1)$ & $1(4.4)$ & \\
\hline Non-smokers & 3847 (77.3) & $48(66.7)$ & & $2199(79.2)$ & $28(58.3)$ & & $1617(75.1)$ & $19(82.6)$ & \\
\hline Total pack-years & $0.7 \pm 3.5$ & $1.4 \pm 4.2$ & 0.22 & $0.7 \pm 3.3$ & $2.1 \pm 5.2$ & 0.13 & $0.6 \pm 3.8$ & $0.2 \pm 0.7$ & 0.03 \\
\hline \multicolumn{10}{|l|}{ Alcohol consumption } \\
\hline Current drinkers & $2300(46.2)$ & $36(50.0)$ & 0.80 & $1524(54.9)$ & $26(54.2)$ & 0.98 & $757(35.1)$ & $9(39.1)$ & 0.62 \\
\hline Ex-drinkers & $188(3.8)$ & $2(2.8)$ & & $108(3.9)$ & $2(4.2)$ & & $79(3.7)$ & $0(0.0)$ & \\
\hline Non-drinkers & $2224(44.7)$ & $31(43.1)$ & & $1048(37.7)$ & $19(39.6)$ & & $1157(53.7)$ & $12(52.2)$ & \\
\hline Total alcohol consumption (g/day) & $17 \pm 64$ & $18 \pm 49$ & 0.93 & $22 \pm 66$ & $20 \pm 55$ & 0.86 & $11 \pm 60$ & $14 \pm 35$ & 0.78 \\
\hline Physical activity (Yes) & $2242(45.1)$ & $35(48.6)$ & 0.86 & 1210 (43.6) & $24(50.0)$ & 0.52 & 1011 (46.9) & $11(47.8)$ & $>0.99$ \\
\hline
\end{tabular}


Table 1. Cont.

\begin{tabular}{|c|c|c|c|c|c|c|c|c|c|}
\hline \multirow{2}{*}{ Characteristics } & \multicolumn{3}{|c|}{ Total $(n=5046)$} & \multicolumn{3}{|c|}{ Premenopausal $(n=2825)$} & \multicolumn{3}{|c|}{ Postmenopausal $(n=2177)$} \\
\hline & Non-Cases $(n=4974)$ & Cases $(n=72)$ & $p$ Value & Non-Cases $(n=2777)$ & Cases $(n=48)$ & $p$ Value & Non-Cases $(n=2154)$ & Cases $(n=23)$ & $p$ Value \\
\hline \multicolumn{10}{|l|}{ Age at menarche (years) } \\
\hline$\leq 13$ & $981(19.7)$ & $13(18.1)$ & 0.88 & $749(27.0)$ & $10(20.8)$ & 0.66 & $228(10.6)$ & $3(13.0)$ & 0.67 \\
\hline 14 & $963(19.4)$ & $16(22.2)$ & & $631(22.7)$ & $14(29.2)$ & & $325(15.1)$ & $2(8.7)$ & \\
\hline 15 & $1111(22.3)$ & $14(19.4)$ & & $651(23.4)$ & $11(22.9)$ & & $450(20.9)$ & $3(13.0)$ & \\
\hline$\geq 16$ & $1587(31.9)$ & $22(30.6)$ & & $625(22.5)$ & $10(20.8)$ & & $955(44.3)$ & $12(52.2)$ & \\
\hline \multicolumn{10}{|l|}{ Parity } \\
\hline 0 & $328(6.6)$ & $7(9.7)$ & 0.20 & $257(9.3)$ & $5(10.4)$ & 0.96 & $69(3.2)$ & $2(8.7)$ & 0.10 \\
\hline 1 & $467(9.4)$ & $7(9.7)$ & & $353(12.7)$ & $6(12.5)$ & & $110(5.1)$ & $1(4.4)$ & \\
\hline 2 & $2587(52.0)$ & $43(59.7)$ & & $1686(60.7)$ & $30(62.5)$ & & $885(41.1)$ & $13(56.5)$ & \\
\hline$\geq 3$ & $1592(32.0)$ & $15(20.8)$ & & $481(17.3)$ & 7 (14.6) & & $1090(50.6)$ & $7(30.4)$ & \\
\hline Lactation (Yes) & 2903 (58.4) & $43(59.7)$ & 0.84 & $1472(53.0)$ & $27(56.3)$ & $>0.99$ & 1412 (65.6) & $16(69.6)$ & 0.25 \\
\hline Oral contraceptive use (Yes) & $864(17.4)$ & $12(16.7)$ & 0.91 & 403 (14.5) & $7(14.6)$ & $>0.99$ & $455(21.1)$ & $5(21.7)$ & $>0.99$ \\
\hline Benign breast tumor history (Yes) & $186(3.7)$ & $7(9.7)$ & 0.02 & $113(4.1)$ & $2(4.2)$ & 0.67 & $71(3.3)$ & $5(21.7)$ & 0.001 \\
\hline Menopausal status (Yes) ${ }^{\mathbf{b}}$ & $2154(43.3)$ & $23(31.9)$ & 0.07 & - & - & - & - & - & - \\
\hline Postmenopausal hormone use (yes) ${ }^{c}$ & - & - & - & - & - & - & $613(28.5)$ & $9(39.1)$ & 0.31 \\
\hline Age at menopause (years) ${ }^{\mathrm{c}}$ & - & - & - & - & - & - & & & \\
\hline$<46$ & - & - & - & - & - & - & $380(17.6)$ & $4(17.4)$ & 0.53 \\
\hline $46-<49$ & - & - & - & - & - & - & $330(15.3)$ & $2(8.7)$ & \\
\hline $49-<52$ & - & - & - & - & - & - & $321(14.9)$ & $6(26.1)$ & \\
\hline$\geq 52$ & - & - & - & - & - & - & $488(22.7)$ & $5(21.7)$ & \\
\hline Type of menopause ${ }^{c}$ & - & - & - & - & - & - & & & \\
\hline Natural & - & - & - & - & - & - & 1485 (68.9) & $17(73.9)$ & 0.84 \\
\hline Surgical, other & - & - & - & - & - & - & $642(29.8)$ & $6(26.1)$ & \\
\hline
\end{tabular}

Missing data are included in the total \%. ${ }^{\text {a }}$ The values are presented as the mean \pm standard deviation (SD) or $n(\%)$ using Student's $t$-test, chi-square test, or Fisher's exact test; ${ }^{\mathrm{b}}$ In all women; ${ }^{\mathrm{c}}$ In postmenopausal women.

Table 2. Sixteen food groups and their associations with the risk of breast cancer in all subjects.

\begin{tabular}{|c|c|c|c|c|c|c|c|c|c|c|c|c|}
\hline \multirow{3}{*}{ Food Group } & \multirow{3}{*}{$\begin{array}{l}\text { Consumption } \\
\text { Frequency a }\end{array}$} & \multicolumn{11}{|c|}{ All Women } \\
\hline & & \multicolumn{2}{|c|}{ All $(n=5046)$} & \multicolumn{2}{|c|}{ Case $(n=72)$} & \multirow{2}{*}{$\begin{array}{l}\text { Person } \\
\text { Years }\end{array}$} & \multicolumn{2}{|c|}{ Age-Adjusted } & \multicolumn{2}{|c|}{ Multivariate $1^{\mathrm{b}}$} & \multicolumn{2}{|c|}{ Multivariate $2^{\mathrm{c}}$} \\
\hline & & $N$ & $\%$ & $n$ & $\%$ & & HR & $95 \% \mathrm{CI}$ & HR & $95 \% \mathrm{CI}$ & HR & $95 \% \mathrm{CI}$ \\
\hline \multirow[b]{2}{*}{ Cereals } & Low & 1738 & 34.4 & 26 & 36.1 & 16,876 & 1.00 & & 1.00 & & 1.00 & \\
\hline & High & 3308 & 65.6 & 46 & 63.9 & 30,837 & 0.96 & $0.59-1.55$ & 0.86 & $0.52-1.40$ & 0.95 & $0.58-1.57$ \\
\hline \multirow{2}{*}{ Salted vegetables and seafood } & Low & 2838 & 56.2 & 41 & 56.9 & 27,009 & 1.00 & & 1.00 & & 1.00 & \\
\hline & High & 2208 & 43.8 & 31 & 43.1 & 20,704 & 0.98 & $0.62-1.57$ & 0.99 & $0.62-1.58$ & 0.98 & $0.61-1.58$ \\
\hline \multirow{2}{*}{ Light-colored vegetables } & Low & 2083 & 41.3 & 32 & 44.4 & 19,610 & 1.00 & & 1.00 & & 1.00 & \\
\hline & High & 2963 & 58.7 & 40 & 55.6 & 28,103 & 0.88 & $0.55-1.40$ & 0.86 & $0.54-1.37$ & 0.87 & $0.54-1.38$ \\
\hline
\end{tabular}


Table 2. Cont

\begin{tabular}{|c|c|c|c|c|c|c|c|c|c|c|c|c|}
\hline \multirow{3}{*}{ Food Group } & \multirow{3}{*}{$\begin{array}{l}\text { Consumption } \\
\text { Frequency a }\end{array}$} & \multicolumn{11}{|c|}{ All Women } \\
\hline & & \multicolumn{2}{|c|}{ All $(n=5046)$} & \multicolumn{2}{|c|}{ Case $(n=72)$} & \multirow{2}{*}{$\begin{array}{c}\text { Person } \\
\text { Years }\end{array}$} & \multicolumn{2}{|c|}{ Age-Adjusted } & \multicolumn{2}{|c|}{ Multivariate $\mathbf{1}^{\mathrm{b}}$} & \multicolumn{2}{|c|}{ Multivariate $2^{\mathrm{C}}$} \\
\hline & & $N$ & $\%$ & $n$ & $\%$ & & HR & $95 \%$ CI & HR & $95 \%$ CI & HR & $95 \% \mathrm{CI}$ \\
\hline \multirow{2}{*}{ Green-yellow vegetables } & Low & 3101 & 61.5 & 38 & 52.8 & 29,564 & 1.00 & \multirow[b]{2}{*}{$0.92-2.32$} & 1.00 & \multirow[b]{2}{*}{$0.87-2.20$} & 1.00 & \multirow[b]{2}{*}{$0.91-2.33$} \\
\hline & High & 1945 & 38.6 & 34 & 47.2 & 18,149 & 1.46 & & 1.38 & & 1.46 & \\
\hline \multirow[b]{2}{*}{ Seaweed } & Low & 1746 & 34.6 & 25 & 34.7 & 16,777 & 1.00 & \multirow[b]{2}{*}{$0.62-1.64$} & 1.00 & \multirow[b]{2}{*}{$0.59-1.57$} & 1.00 & \multirow[b]{2}{*}{$0.65-1.73$} \\
\hline & High & 3300 & 65.4 & 47 & 65.3 & 30,936 & 1.01 & & 0.96 & & 1.06 & \\
\hline \multirow[b]{2}{*}{ Fruit } & Low & 2554 & 50.6 & 35 & 48.6 & 24,168 & 1.00 & \multirow[b]{2}{*}{$0.69-1.73$} & 1.00 & \multirow[b]{2}{*}{$0.65-1.70$} & 1.00 & \multirow[b]{2}{*}{$0.76-1.97$} \\
\hline & High & 2492 & 49.4 & 37 & 51.4 & 23,545 & 1.09 & & 1.05 & & 1.22 & \\
\hline \multirow{2}{*}{ Grilled meat } & Low & 2908 & 57.6 & 31 & 43.1 & 27,398 & 1.00 & \multirow[b]{2}{*}{$1.12-2.91$} & 1.00 & \multirow[b]{2}{*}{$1.03-2.68$} & 1.00 & \multirow[b]{2}{*}{$1.09-2.85$} \\
\hline & High & 2138 & 42.4 & 41 & 56.9 & 20,315 & 1.80 & & 1.66 & & 1.77 & \\
\hline \multirow{2}{*}{ Healthy protein foods } & Low & 2885 & 57.2 & 35 & 48.6 & 27,391 & 1.00 & \multirow[b]{2}{*}{$0.89-2.25$} & 1.00 & \multirow[b]{2}{*}{$0.78-2.00$} & 1.00 & \\
\hline & High & 2161 & 42.8 & 37 & 51.4 & 20,322 & 1.42 & & 1.25 & & 1.46 & $0.91-2.34$ \\
\hline & Low & 2864 & 56.8 & 36 & 50.0 & 27,142 & 1.00 & & 1.00 & & 1.00 & \\
\hline Darry foods & High & 2182 & 43.2 & 36 & 50.0 & 20,571 & 1.33 & $0.83-2.10$ & 1.24 & $0.78-1.97$ & 1.32 & $0.83-2.11$ \\
\hline & Low & 2293 & 45.4 & 31 & 43.1 & 21,766 & 1.00 & & 1.00 & & 1.00 & \\
\hline Bony fish & High & 2753 & 54.6 & 41 & 56.9 & 25,947 & 1.12 & $0.70-1.80$ & 1.10 & $0.69-1.78$ & 1.14 & $0.71-1.83$ \\
\hline & Low & 2599 & 51.5 & 34 & 47.2 & 24,588 & 1.00 & & 1.00 & & 1.00 & \\
\hline Fried foods & High & 2447 & 48.5 & 38 & 52.8 & 23,125 & 1.18 & $0.73-1.89$ & 1.06 & $0.66-1.71$ & 1.19 & $0.74-1.92$ \\
\hline & Low & 2153 & 42.7 & 22 & 30.6 & 20,527 & 1.00 & & 1.00 & & 1.00 & \\
\hline High-cholesterol foods & High & 2893 & 57.3 & 50 & 69.4 & 27,186 & 1.71 & $1.03-2.85$ & 1.48 & $0.88-2.48$ & 1.69 & $1.01-2.82$ \\
\hline & Low & 3143 & 62.3 & 43 & 59.7 & 29,641 & 1.00 & & 1.00 & & 1.00 & \\
\hline Animal fat-rich foods & High & 1903 & 37.7 & 29 & 40.3 & 18,072 & 1.09 & $0.68-1.77$ & 1.03 & $0.63-1.67$ & 1.05 & $0.64-1.71$ \\
\hline & Low & 2180 & 43.2 & 32 & 44.4 & 20,395 & 1.00 & & 1.00 & & 1.00 & \\
\hline Sweet foods & High & 2866 & 56.8 & 40 & 55.6 & 27,318 & 0.92 & $0.57-1.47$ & 0.82 & $0.51-1.33$ & 0.90 & $0.56-1.45$ \\
\hline & Low & 3152 & 62.5 & 42 & 58.3 & 29,741 & 1.00 & & 1.00 & & 1.00 & \\
\hline Fast-foods & High & 1894 & 37.5 & 30 & 41.7 & 17,972 & 1.17 & 0.71-1.94 & 1.03 & $0.62-1.70$ & 1.16 & $0.70-1.90$ \\
\hline & Low & 2128 & 42.2 & 30 & 41.7 & 20,133 & 1.00 & & 1.00 & & 1.00 & \\
\hline Cafteinated drinks & High & 2918 & 57.8 & 42 & 58.3 & 27,580 & 1.01 & $0.63-1.62$ & 0.88 & $0.55-1.42$ & 0.90 & $0.55-1.46$ \\
\hline
\end{tabular}

Abbreviations are as follows: $\mathrm{CI}$, confidence interval; HR, hazard ratio. ${ }^{a}$ Cereals/Salted vegetables and seafood: low $\leq$ once a day, high $\geq 2$ times a day. Light-colored vegetables/Caffeinated drink/Fruit: low $\leq 4-6$ times a week, high $\geq$ once a day. Green-yellow vegetables/Healthy protein foods/Dairy foods: low $\leq 2-3$ times a week, high $\geq 4-6$ times a week. Seaweed/Bony fish: low $\leq$ once a week, high $\geq 2-3$ times a week. Grilled meat/High-cholesterol foods/Fast-foods: low $\leq$ once a month, high $\geq 2-3$ times a month. Fried foods/Animal fat-rich foods/Sweet foods:

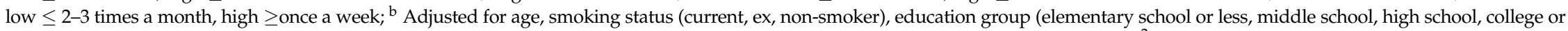
higher), breast benign tumor history (yes, no); ${ }^{c}$ Adjusted for diverse potential confounders: total: age, BMI $\left(<23.0,23.0\right.$ to $\left.<25.0, \geq 25 \mathrm{~kg} / \mathrm{m}^{2}\right)$, family history of breast cancer (yes, no), smoking status (current, ex, non-smoker), alcohol consumption (current, ex, non-drinker), physical activity (yes, no), age at menarche $(\leq 13,14,15, \geq 16$ years), parity $(0,1,2, \geq 3)$, oral contraceptive use (yes, no), benign breast tumor history (yes, no), hormone use and menopausal status (premenopausal, postmenopausal users, postmenopausal nonusers, unknown menopausal status), and age at menopause (premenopausal, menopause at $<46,46-48,49-51, \geq 52$ years, unknown menopausal status). 
Table 3. Sixteen food groups and their associations with the risk of breast cancer in premenopausal women.

\begin{tabular}{|c|c|c|c|c|c|c|c|c|c|c|c|c|}
\hline \multirow{3}{*}{ Food Group } & \multirow{3}{*}{$\begin{array}{l}\text { Consumption } \\
\text { Frequency }{ }^{a}\end{array}$} & \multicolumn{11}{|c|}{ Premenopausal Women } \\
\hline & & \multicolumn{2}{|c|}{ All $(n=2825)$} & \multicolumn{2}{|c|}{ Case $(n=48)$} & \multirow{2}{*}{$\begin{array}{l}\text { Person } \\
\text { Years }\end{array}$} & \multicolumn{2}{|c|}{ Age-Adjusted } & \multicolumn{2}{|c|}{ Multivariate $\mathbf{1}^{\mathrm{b}}$} & \multicolumn{2}{|c|}{ Multivariate $2^{\mathrm{C}}$} \\
\hline & & $N$ & $\%$ & $n$ & $\%$ & & HR & $95 \% \mathrm{CI}$ & HR & $95 \% \mathrm{CI}$ & HR & $95 \% \mathrm{CI}$ \\
\hline \multirow[b]{2}{*}{ Cereals } & Low & 929 & 32.9 & 15 & 31.3 & 9054 & 1.00 & \multirow[b]{2}{*}{$0.63-2.13$} & 1.00 & \multirow[b]{2}{*}{$0.64-2.20$} & 1.00 & \multirow[b]{2}{*}{$0.61-2.17$} \\
\hline & High & 1896 & 67.1 & 33 & 68.8 & 17,666 & 1.15 & & 1.19 & & 1.15 & \\
\hline \multirow{2}{*}{ Salted vegetables and seafood } & Low & 1560 & 55.2 & 25 & 52.1 & 14,877 & 1.00 & \multirow[b]{2}{*}{$0.66-2.06$} & 1.00 & \multirow[b]{2}{*}{$0.68-2.13$} & 1.00 & \multirow[b]{2}{*}{$0.65-2.09$} \\
\hline & High & 1265 & 44.8 & 23 & 47.9 & 11,843 & 1.17 & & 1.21 & & 1.17 & \\
\hline \multirow{2}{*}{ Light-colored vegetables } & Low & 1168 & 41.4 & 25 & 52.1 & 10,971 & 1.00 & \multirow[b]{2}{*}{$0.36-1.10$} & 1.00 & \multirow[b]{2}{*}{$0.36-1.12$} & 1.00 & \multirow[b]{2}{*}{$0.35-1.09$} \\
\hline & High & 1657 & 58.7 & 23 & 47.9 & 15,749 & 0.63 & & 0.64 & & 0.61 & \\
\hline \multirow{2}{*}{ Green-yellow vegetables } & Low & 1792 & 63.4 & 27 & 56.3 & 17,071 & 1.00 & \multirow[b]{2}{*}{$0.77-2.41$} & 1.00 & \multirow[b]{2}{*}{$0.78-2.44$} & 1.00 & \multirow[b]{2}{*}{$0.75-2.36$} \\
\hline & High & 1033 & 36.6 & 21 & 43.8 & 9649 & 1.36 & & 1.38 & & 1.33 & \\
\hline & Low & 940 & 33.3 & 19 & 39.6 & 9054 & 1.00 & & 1.00 & & 1.00 & \\
\hline Seaweed & High & 1885 & 66.7 & 29 & 60.4 & 17,666 & 0.78 & $0.44-1.40$ & 0.81 & $0.45-1.44$ & 0.76 & $0.42-1.38$ \\
\hline & Low & 1497 & 53.0 & 24 & 50.0 & 14,108 & 1.00 & & 1.00 & & 1.00 & \\
\hline Fruit & High & 1328 & 47.0 & 24 & 50.0 & 12,612 & 1.10 & $0.62-1.94$ & 1.25 & $0.71-2.22$ & 1.23 & $0.69-2.20$ \\
\hline & Low & 1413 & 50.0 & 21 & 43.8 & 13,287 & 1.00 & & 1.00 & & 1.00 & \\
\hline Grilled meat & High & 1412 & 50.0 & 27 & 56.3 & 13,433 & 1.36 & $0.77-2.42$ & 1.33 & $0.75-2.36$ & 1.36 & $0.77-2.43$ \\
\hline Healthy nrotein foods & Low & 1582 & 56.0 & 26 & 54.2 & 15,028 & 1.00 & & 1.00 & & 1.00 & \\
\hline Healthy protenn foods & High & 1243 & 44.0 & 22 & 45.8 & 11,692 & 1.08 & $0.61-1.91$ & 1.12 & $0.63-1.97$ & 1.12 & $0.63-2.00$ \\
\hline & Low & 1666 & 59.0 & 26 & 54.2 & 15,786 & 1.00 & & 1.00 & & 1.00 & \\
\hline Dairy food & High & 1159 & 41.0 & 22 & 45.8 & 10,934 & 1.21 & $0.69-2.14$ & 1.22 & $0.69-2.16$ & 1.20 & $0.67-2.13$ \\
\hline Bony fish & Low & 1391 & 49.2 & 24 & 50.0 & 13,246 & 1.00 & & 1.00 & & 1.00 & \\
\hline & High & 1434 & 50.8 & 24 & 50.0 & 13,474 & 0.92 & $0.52-1.63$ & 0.96 & $0.54-1.70$ & 0.95 & $0.53-1.69$ \\
\hline & Low & 1222 & 43.3 & 22 & 45.8 & 11,554 & 1.00 & & 1.00 & & 1.00 & \\
\hline Fried foods & High & 1603 & 56.7 & 26 & 54.2 & 15,166 & 0.97 & $0.55-1.73$ & 1.00 & $0.56-1.78$ & 1.00 & $0.56-1.79$ \\
\hline & Low & 1014 & 35.9 & 14 & 29.2 & 9707 & 1.00 & & 1.00 & & 1.00 & \\
\hline High-cholesterol foods & High & 1811 & 64.1 & 34 & 70.8 & 17,013 & 1.45 & $0.78-2.70$ & 1.46 & $0.78-2.73$ & 1.42 & $0.75-2.67$ \\
\hline & Low & 1519 & 53.8 & 27 & 56.3 & 14,284 & 1.00 & & 1.00 & & 1.00 & \\
\hline Animal tat-rich foods & High & 1306 & 46.2 & 21 & 43.8 & 12,436 & 0.94 & $0.53-1.67$ & 0.93 & $0.52-1.64$ & 0.93 & $0.52-1.67$ \\
\hline & Low & 1036 & 36.7 & 18 & 37.5 & 9633 & 1.00 & & 1.00 & & 1.00 & \\
\hline Sweet foods & High & 1789 & 63.3 & 30 & 62.5 & 17,087 & 1.01 & $0.56-1.82$ & 1.07 & $0.59-1.94$ & 1.02 & $0.56-1.86$ \\
\hline & Low & 1422 & 50.3 & 25 & 52.1 & 13,376 & 1.00 & & 1.00 & & 1.00 & \\
\hline Fast-foods & High & 1403 & 49.7 & 23 & 47.9 & 13,344 & 1.03 & $0.58-1.85$ & 1.07 & $0.60-1.92$ & 1.05 & $0.58-1.89$ \\
\hline Caffeinated drinks & Low & 995 & 35.2 & 16 & 33.3 & 9373 & 1.00 & & 1.00 & & 1.00 & \\
\hline Cafteinated drinks & High & 1830 & 64.8 & 32 & 66.7 & 17,347 & 1.08 & $0.59-1.97$ & 1.06 & $0.58-1.93$ & 1.07 & $0.58-1.96$ \\
\hline
\end{tabular}

Abbreviations are as follows: CI, confidence interval; HR, hazard ratio. ${ }^{a}$ Cereals/Salted vegetables and seafood: low $\leq$ once a day, high $\geq 2$ times a day. Light-colored vegetables/Caffeinated drinks/Fruit: low $\leq 4-6$ times a week, high $\geq$ once a day. Green-yellow vegetables/Healthy protein food/Dairy food: low $\leq 2-3$ times a week, high $\geq 4-6$ times a week. Seaweed/Bony fish: low $\leq$ once a week, high $\geq 2-3$ times a week. Grilled meat/High-cholesterol foods/Fast-foods: low $\leq$ once a month, high $\geq 2-3$ times a month. Fried foods/Animal fat-rich foods/Sweet foods: low $<2-3$ times a month, high $>$ once a week; ${ }^{\mathrm{b}}$ Adjusted for age, smoking status (current, ex, non-smoker); ${ }^{\mathrm{c}}$ Adjusted for diverse potential confounders: total: age, BMI ( $<23.0,23.0$ to $<25.0$, $\geq 25 \mathrm{~kg} / \mathrm{m}^{2}$ ), family history of breast cancer (yes, no), smoking status (current, ex, non-smoker), alcohol consumption (current, ex, non-drinker), physical activity (yes, no), age at menarche ( $\leq 13$, $14,15, \geq 16$ years), parity $(0,1,2, \geq 3)$, oral contraceptive use (yes, no), and benign breast tumor history (yes, no). 
Table 4. Sixteen food groups and their associations with the risk of breast cancer in postmenopausal women.

\begin{tabular}{|c|c|c|c|c|c|c|c|c|c|c|c|c|}
\hline \multirow{3}{*}{ Food Group } & \multirow{3}{*}{$\begin{array}{l}\text { Consumption } \\
\text { Frequency }^{a}\end{array}$} & \multicolumn{11}{|c|}{ Postmenopausal Women } \\
\hline & & \multicolumn{2}{|c|}{ All $(n=2177)$} & \multicolumn{2}{|c|}{ Case $(n=23)$} & \multirow{2}{*}{$\begin{array}{l}\text { Person } \\
\text { Years }\end{array}$} & \multicolumn{2}{|c|}{ Age-Adjusted } & \multicolumn{2}{|c|}{ Multivariate $\mathbf{1}^{\mathrm{b}}$} & \multicolumn{2}{|c|}{ Multivariate $2^{\mathrm{c}}$} \\
\hline & & $N$ & $\%$ & $n$ & $\%$ & & HR & $95 \% \mathrm{CI}$ & HR & $95 \% \mathrm{CI}$ & HR & $95 \% \mathrm{CI}$ \\
\hline \multirow{2}{*}{ Cereals } & Low & 786 & 36.1 & 11 & 47.8 & 7593 & 1.00 & \multirow[b]{2}{*}{$0.28-1.41$} & 1.00 & \multirow[b]{2}{*}{$0.24-1.23$} & 1.00 & \multirow[b]{2}{*}{$0.22-1.25$} \\
\hline & High & 1391 & 63.9 & 12 & 52.2 & 12,986 & 0.62 & & 0.54 & & 0.53 & \\
\hline \multirow{2}{*}{ Salted vegetables and seafood } & Low & 1254 & 57.6 & 16 & 69.6 & 11,894 & 1.00 & \multirow[b]{2}{*}{$0.24-1.44$} & 1.00 & \multirow[b]{2}{*}{$0.24-1.42$} & 1.00 & \multirow[b]{2}{*}{$0.18-1.14$} \\
\hline & High & 923 & 42.4 & 7 & 30.4 & 8685 & 0.59 & & 0.59 & & 0.45 & \\
\hline \multirow{2}{*}{ Light-colored vegetables } & Low & 894 & 41.1 & 7 & 30.4 & 8437 & 1.00 & \multirow[b]{2}{*}{$0.65-3.86$} & 1.00 & \multirow[b]{2}{*}{$0.56-3.36$} & 1.00 & \multirow[b]{2}{*}{$0.55-3.3$} \\
\hline & High & 1283 & 58.9 & 16 & 69.6 & 12,142 & 1.59 & & 1.38 & & 1.37 & \\
\hline \multirow{2}{*}{ Green-yellow vegetables } & Low & 1280 & 58.8 & 11 & 47.8 & 12,215 & 1.00 & \multirow[b]{2}{*}{$0.70-3.57$} & 1.00 & \multirow[b]{2}{*}{$0.59-3.07$} & 1.00 & \multirow[b]{2}{*}{$0.62-3.3$} \\
\hline & High & 897 & 41.2 & 12 & 52.2 & 8364 & 1.58 & & 1.34 & & 1.42 & \\
\hline & Low & 790 & 36.3 & 6 & 26.1 & 7566 & 1.00 & & 1.00 & & 1.00 & \\
\hline Seaweed & High & 1387 & 63.7 & 17 & 73.9 & 13,013 & 1.63 & $0.64-4.15$ & 1.40 & $0.55-3.59$ & 1.73 & $0.67-4.50$ \\
\hline & Low & 1035 & 47.5 & 10 & 43.5 & 9859 & 1.00 & & 1.00 & & 1.00 & \\
\hline Fruit & High & 1142 & 52.5 & 13 & 56.5 & 10,720 & 1.18 & $0.52-2.70$ & 0.86 & $0.36-2.03$ & 1.22 & $0.51-2.92$ \\
\hline & Low & 1475 & 67.8 & 10 & 43.5 & 13,912 & 1.00 & & 1.00 & & 1.00 & \\
\hline Grilled meat & High & 702 & 32.3 & 13 & 56.5 & 6667 & 2.80 & $1.22-6.41$ & 2.41 & $1.05-5.54$ & 3.06 & $1.31-7.15$ \\
\hline Healthy protein foods & Low & 1275 & 58.6 & 9 & 39.1 & 12,093 & 1.00 & & 1.00 & & 1.00 & \\
\hline Healtny protein foods & High & 902 & 41.4 & 14 & 60.9 & 8486 & 2.23 & $0.96-5.15$ & 1.77 & $0.74-4.20$ & 2.28 & $0.94-5.52$ \\
\hline & Low & 1175 & 54.0 & 10 & 43.5 & 11,130 & 1.00 & & 1.00 & & 1.00 & \\
\hline Dairy foods & High & 1002 & 46.0 & 13 & 56.5 & 9449 & 1.53 & $0.67-3.49$ & 1.31 & $0.57-3.01$ & 1.56 & $0.67-3.65$ \\
\hline & Low & 882 & 40.5 & 7 & 30.4 & 8324 & 1.00 & & 1.00 & & 1.00 & \\
\hline Bony fish & High & 1295 & 59.5 & 16 & 69.6 & 12,255 & 1.53 & $0.63-3.72$ & 1.34 & $0.54-3.29$ & 1.38 & $0.55-3.46$ \\
\hline & Low & 1351 & 62.1 & 11 & 47.8 & 12,790 & 1.00 & & 1.00 & & 1.00 & \\
\hline Fried foods & High & 826 & 37.9 & 12 & 52.2 & 7789 & 1.84 & $0.81-4.17$ & 1.47 & $0.64-3.38$ & 1.78 & $0.75-4.21$ \\
\hline High-cholesterol foods & Low & 1124 & 51.6 & 8 & 34.8 & 10,677 & 1.00 & & 1.00 & & 1.00 & \\
\hline Hign-cnoresterol roods & High & 1053 & 48.4 & 15 & 65.2 & 9902 & 2.06 & $0.87-4.88$ & 1.59 & $0.66-3.82$ & 1.97 & $0.81-4.80$ \\
\hline & Low & 1595 & 73.3 & 16 & 69.6 & 15,080 & 1.00 & & 1.00 & & 1.00 & \\
\hline Animal fat-rich foods & High & 582 & 26.7 & 7 & 30.4 & 5499 & 1.25 & $0.51-3.07$ & 1.11 & $0.45-2.70$ & 1.18 & $0.47-2.99$ \\
\hline & Lo & 1121 & 51.5 & 13 & 56.5 & 10,552 & 1.00 & & 1.00 & & 1.00 & \\
\hline Sweet foods & High & 1056 & 48.5 & 10 & 43.5 & 10,027 & 0.83 & $0.36-1.90$ & 0.69 & $0.30-1.60$ & 0.66 & $0.27-1.57$ \\
\hline & & 1699 & 78.0 & 16 & 69.6 & 16,078 & 1.00 & & 1.00 & & 1.00 & \\
\hline Fast-foods & High & 478 & 22.0 & 7 & 30.4 & 4501 & 1.68 & $0.68-4.14$ & 1.29 & $0.52-3.20$ & 1.47 & $0.58-3.71$ \\
\hline Caffeinated drinks & Low & 1118 & 51.4 & 14 & 60.9 & 10,617 & 1.00 & & 1.00 & & 1.00 & \\
\hline Caffeinated drinks & High & 1059 & 48.6 & 9 & 39.1 & 9962 & 0.70 & $0.30-1.64$ & 0.61 & $0.26-1.42$ & 0.56 & $0.23-1.35$ \\
\hline
\end{tabular}

Abbreviations are as follows: $\mathrm{CI}$, confidence interval; HR, hazard ratio. ${ }^{a}$ Cereals/Salted vegetables and seafood: low $\leq$ once a day, high $\geq 2$ times a day. Light-colored vegetables/Caffeinated drinks/Fruit: low $<4-6$ times a week, high $>$ once a day. Green-yellow vegetables/Healthy protein foods/Dairy foods: low $\leq 2-3$ times a week, high $>4-6$ times a week. Seaweed/Bony fish: low $\leq$ once a week, high $\geq 2-3$ times a week. Grilled meat/High-cholesterol foods/Fast-foods: low $\leq$ once a month, high $\geq 2-3$ times a month. Fried foods/Animal fat-rich foods/Sweet foods: low $\leq 2-3$ times a month, high $\geq$ once a week; ${ }^{b}$ Adjusted for age, education group (elementary school or less, middle school, high school, college or higher), breast benign tumor history (yes, no); ${ }^{c}$ Adjusted for diverse potential confounders: total: age, BMI ( $<23.0,23.0$ to $<25.0, \geq 25 \mathrm{~kg} / \mathrm{m}^{2}$ ), family history of breast cancer (yes, no), smoking status (current, ex, non-smoker), alcohol consumption (current, ex, non-drinker), physical activity (yes, no), age at menarche ( $\leq 13,14,15, \geq 16$ years), parity $(0,1,2, \geq 3)$, oral contraceptive use (yes, no), benign breast tumor history (yes, no), hormone use (yes, no), and age at menopause $(<46,46-48,49-51, \geq 52$ years) 
Table 5. Six dietary habits and their associations with breast cancer risk in all subjects.

\begin{tabular}{|c|c|c|c|c|c|c|c|c|c|c|c|c|}
\hline \multirow{3}{*}{ Dietary Habit } & \multirow{3}{*}{ Frequency } & \multicolumn{11}{|c|}{ All Women } \\
\hline & & \multicolumn{2}{|c|}{ All $(n=5046)$} & \multicolumn{2}{|c|}{ Case $(n=72)$} & \multirow{2}{*}{$\begin{array}{c}\text { Person } \\
\text { Years }\end{array}$} & \multicolumn{2}{|c|}{ Age-Adjusted } & \multicolumn{2}{|c|}{ Multivariate $\mathbf{1}^{\mathrm{b}}$} & \multicolumn{2}{|c|}{ Multivariate $2^{\mathrm{c}}$} \\
\hline & & $N$ & $\%$ & $n$ & $\%$ & & HR & $95 \% \mathrm{CI}$ & HR & $95 \%$ CI & HR & $95 \% \mathrm{CI}$ \\
\hline \multirow{4}{*}{ Meal frequency } & 3 times/day & 3285 & 65.1 & 37 & 51.4 & 31,174 & 1.00 & & 1.00 & & 1.00 & \\
\hline & 2 times/day & 1110 & 22.0 & 18 & 25.0 & 10,327 & 1.49 & $0.84-2.63$ & 1.37 & $0.77-2.44$ & 1.36 & $0.76-2.42$ \\
\hline & Irregular ${ }^{\mathrm{a}}$ & 616 & 12.2 & 17 & 23.6 & 5896 & 2.47 & $1.38-4.41$ & 2.37 & $1.31-4.27$ & 2.19 & $1.20-3.98$ \\
\hline & $p$ for trend & & & & & & 0.003 & & 0.006 & & 0.01 & \\
\hline \multirow{4}{*}{ Breakfast frequency } & Always & 2792 & 55.3 & 31 & 43.1 & 26,361 & 1.00 & & 1.00 & & 1.00 & \\
\hline & Often & 1579 & 31.3 & 29 & 40.3 & 14,992 & 1.67 & $1.00-2.80$ & 1.55 & $0.92-2.60$ & 1.56 & $0.93-2.63$ \\
\hline & Not at all & 657 & 13.0 & 12 & 16.7 & 6193 & 1.68 & $0.85-3.33$ & 1.41 & $0.71-2.84$ & 1.43 & $0.71-2.90$ \\
\hline & $p$ for trend & & & & & & 0.06 & & 0.18 & & 0.17 & \\
\hline \multirow{4}{*}{ Meal time regularity } & Regular & 1969 & 39.0 & 36 & 50.0 & 18,474 & 1.00 & & 1.00 & & 1.00 & \\
\hline & Often & 2549 & 50.5 & 25 & 34.7 & 24,404 & 0.52 & $0.31-0.87$ & 0.53 & $0.32-0.90$ & 0.52 & $0.31-0.88$ \\
\hline & Irregular & 510 & 10.1 & 11 & 15.3 & 4661 & 1.18 & $0.59-2.34$ & 1.24 & $0.62-2.51$ & 1.02 & $0.50-2.06$ \\
\hline & $p$ for trend & & & & & & 0.40 & & 0.48 & & 0.29 & \\
\hline \multirow{4}{*}{ Meal speed } & Slow & 557 & 11.0 & 7 & 9.7 & 5240 & 1.00 & & 1.00 & & 1.00 & \\
\hline & Average & 2634 & 52.2 & 40 & 55.6 & 24,817 & 1.22 & $0.55-2.72$ & 1.41 & $0.63-3.16$ & 1.35 & $0.60-3.04$ \\
\hline & Fast & 1850 & 36.7 & 25 & 34.7 & 17,611 & 1.07 & $0.47-2.49$ & 1.27 & $0.55-2.95$ & 1.17 & $0.50-2.74$ \\
\hline & $p$ for trend & & & & & & 0.91 & & 0.81 & & 0.99 & \\
\hline \multirow{4}{*}{ Overeating } & Less than once a week & 1686 & 33.4 & 19 & 26.4 & 15,817 & 1.00 & & 1.00 & & 1.00 & \\
\hline & 2-3 times a week & 2864 & 56.8 & 43 & 59.7 & 27,211 & 1.26 & $0.74-2.14$ & 1.22 & $0.72-2.08$ & 1.24 & $0.72-2.12$ \\
\hline & More than 4 times a week & 479 & 9.5 & 9 & 12.5 & 4525 & 1.58 & $0.72-3.48$ & 1.57 & $0.71-3.48$ & 1.46 & $0.64-3.31$ \\
\hline & $p$ for trend & & & & & & 0.20 & & 0.22 & & 0.26 & \\
\hline \multirow{4}{*}{ Added spices } & Often & 598 & 11.9 & 10 & 13.9 & 5701 & 1.00 & & 1.00 & & 1.00 & \\
\hline & Sometimes & 2684 & 53.2 & 47 & 65.3 & 25,530 & 1.08 & $0.54-2.13$ & 1.04 & $0.52-2.06$ & 1.06 & $0.53-2.12$ \\
\hline & Never & 1745 & 34.6 & 15 & 20.8 & 16,305 & 0.53 & $0.24-1.19$ & 0.50 & $0.22-1.11$ & 0.54 & $0.24-1.20$ \\
\hline & $p$ for trend & & & & & & 0.04 & & 0.03 & & 0.045 & \\
\hline
\end{tabular}

Abbreviations are as follows: CI, confidence interval; HR, hazard ratio. ${ }^{a}$ Less than one time a day; ${ }^{\mathrm{b}}$ Adjusted for age, education group (elementary school or less, middle school, high school, college or higher), and breast benign tumor history (yes, no); ${ }^{\mathrm{c}}$ Adjusted for diverse potential confounders: total: age, BMI $\left(<23.0,23.0\right.$ to $\left.<25.0, \geq 25 \mathrm{~kg} / \mathrm{m}^{2}\right)$, family history of breast cancer (yes, no), smoking status (current, ex, non-smoker), alcohol consumption (current, ex, non-drinker), physical activity (yes, no), age at menarche ( $\leq 13,14,15, \geq 16$ years), parity $(0,1$, $2, \geq 3$ ), oral contraceptive use (yes, no), benign breast tumor history (yes, no), hormone use and menopausal status (premenopausal, postmenopausal hormone users, postmenopausal

hormone non-users, unknown menopausal status), and age at menopause (premenopausal, menopause at $<46,46-48,49-51$, $\geq 52$ years, unknown menopausal status). 
Table 6. Six dietary habits and their associations with breast cancer risk in premenopausal women.

\begin{tabular}{|c|c|c|c|c|c|c|c|c|c|c|c|c|}
\hline \multirow{3}{*}{ Dietary Habit } & \multirow{3}{*}{ Frequency } & \multicolumn{11}{|c|}{ Premenopausal Women } \\
\hline & & \multicolumn{2}{|c|}{ All $(n=2825)$} & \multicolumn{2}{|c|}{ Case $(n=48)$} & \multirow{2}{*}{$\begin{array}{l}\text { Person } \\
\text { Years }\end{array}$} & \multicolumn{2}{|c|}{ Age-Adjusted } & \multicolumn{2}{|c|}{ Multivariate $\mathbf{1}^{\mathrm{b}}$} & \multicolumn{2}{|c|}{ Multivariate $2^{c}$} \\
\hline & & $N$ & $\%$ & $n$ & $\%$ & & HR & $95 \% \mathrm{CI}$ & HR & $95 \% \mathrm{CI}$ & HR & $95 \% \mathrm{CI}$ \\
\hline \multirow{4}{*}{ Meal frequency } & 3 times/day & 1705 & 60.4 & 23 & 47.9 & 16,191 & 1.00 & & 1.00 & & 1.00 & \\
\hline & 2 times/day & 702 & 24.9 & 13 & 27.1 & 6527 & 1.50 & $0.76-2.96$ & 1.31 & $0.66-2.61$ & 1.34 & $0.67-2.68$ \\
\hline & Irregular ${ }^{a}$ & 400 & 14.2 & 12 & 25.0 & 3838 & 2.34 & $1.16-4.71$ & 2.15 & $1.06-4.40$ & 2.35 & $1.13-4.91$ \\
\hline & $p$ for trend & & & & & & 0.02 & & 0.044 & & 0.03 & \\
\hline \multirow{4}{*}{ Breakfast frequency } & Always & 1340 & 47.4 & 20 & 41.7 & 12,623 & 1.00 & & 1.00 & & 1.00 & \\
\hline & Often & 1011 & 35.8 & 18 & 37.5 & 9604 & 1.25 & $0.66-2.36$ & 1.16 & $0.61-2.20$ & 1.17 & $0.62-2.24$ \\
\hline & Not at all & 466 & 16.5 & 10 & 20.8 & 4414 & 1.57 & $0.73-3.38$ & 1.33 & $0.61-2.92$ & 1.37 & $0.62-3.05$ \\
\hline & $p$ for trend & & & & & & 0.24 & & 0.47 & & 0.45 & \\
\hline \multirow{4}{*}{ Meal time regularity } & Regular & 981 & 34.7 & 23 & 47.9 & 9162 & 1.00 & & 1.00 & & 1.00 & \\
\hline & Often & 1487 & 52.6 & 18 & 37.5 & 14,284 & 0.53 & $0.28-0.98$ & 0.51 & $0.27-0.94$ & 0.52 & $0.28-0.98$ \\
\hline & Irregular & 348 & 12.3 & 7 & 14.6 & 3187 & 0.93 & $0.40-2.17$ & 0.76 & $0.32-1.79$ & 0.76 & $0.31-1.83$ \\
\hline & $p$ for trend & & & & & & 0.30 & & 0.17 & & 0.19 & \\
\hline \multirow{4}{*}{ Meal speed } & Slow & 317 & 11.2 & 2 & 4.2 & 3025 & 1.00 & & 1.00 & & 1.00 & \\
\hline & Average & 1501 & 53.1 & 29 & 60.4 & 14,138 & 3.03 & $0.72-12.71$ & 3.28 & $0.78-13.77$ & 3.28 & $0.78-13.84$ \\
\hline & Fast & 1005 & 35.6 & 17 & 35.4 & 9536 & 2.62 & $0.61-11.35$ & 2.75 & $0.63-11.93$ & 2.94 & $0.67-12.86$ \\
\hline & $p$ for trend & & & & & & 0.49 & & 0.49 & & 0.38 & \\
\hline \multirow{4}{*}{ Overeating } & Less than once a week & 884 & 31.3 & 13 & 27.1 & 8257 & 1.00 & & 1.00 & & 1.00 & \\
\hline & 2-3 times a week & 1627 & 57.6 & 28 & 58.3 & 15,466 & 1.18 & $0.61-2.27$ & 1.12 & $0.58-2.17$ & 1.18 & $0.61-2.30$ \\
\hline & More than 4 times a week & 304 & 10.8 & 7 & 14.6 & 2895 & 1.61 & $0.64-4.04$ & 1.37 & $0.54-3.47$ & 1.60 & $0.62-4.15$ \\
\hline & $p$ for trend & & & & & & 0.35 & & 0.54 & & 0.37 & \\
\hline \multirow{4}{*}{ Added spices } & Often & 304 & 10.8 & 8 & 16.7 & 2854 & 1.00 & & 1.00 & & 1.00 & \\
\hline & Sometimes & 1479 & 52.4 & 29 & 60.4 & 14,101 & 0.75 & $0.34-1.63$ & 0.82 & $0.37-1.80$ & 0.78 & $0.35-1.74$ \\
\hline & Never & 1037 & 36.7 & 11 & 22.9 & 9719 & 0.41 & $0.16-1.01$ & 0.46 & $0.19-1.16$ & 0.43 & $0.17-1.08$ \\
\hline & $p$ for trend & & & & & & 0.03 & & 0.06 & & 0.045 & \\
\hline
\end{tabular}

Abbreviations are as follows: $\mathrm{CI}$, confidence interval; HR, hazard ratio. ${ }^{a}$ Less than one time a day; ${ }^{\mathrm{b}}$ Adjusted for age, education group (elementary school or less, middle school, high school, college or higher), and breast benign tumor history (yes, no); ${ }^{\mathrm{c}}$ Adjusted for diverse potential confounders: total: age, BMI $\left(<23.0,23.0\right.$ to $\left.<25.0, \geq 25 \mathrm{~kg} / \mathrm{m}^{2}\right)$, family history of breast

cancer (yes, no), smoking status (current, ex, non-smoker), alcohol consumption (current, ex, non-drinker), physical activity (yes, no), age at menarche ( $\leq 13,14,15, \geq 16$ years), parity $(0,1$,

$2, \geq 3$ ), oral contraceptive use (yes, no), and benign breast tumor history (yes, no). 
Table 7. Six dietary habits and their associations with breast cancer risk in postmenopausal women.

\begin{tabular}{|c|c|c|c|c|c|c|c|c|c|c|c|c|}
\hline \multirow{3}{*}{ Dietary Habit } & \multirow{3}{*}{ Frequency } & \multicolumn{11}{|c|}{ Postmenopausal Women } \\
\hline & & \multicolumn{2}{|c|}{ All $(n=2177)$} & \multicolumn{2}{|c|}{ Case $(n=23)$} & \multirow{2}{*}{$\begin{array}{c}\text { Person } \\
\text { Years }\end{array}$} & \multicolumn{2}{|c|}{ Age-Adjusted } & \multicolumn{2}{|c|}{ Multivariate $1^{b}$} & \multicolumn{2}{|c|}{ Multivariate $2^{\mathrm{c}}$} \\
\hline & & $N$ & $\%$ & $n$ & $\%$ & & HR & $95 \% \mathrm{CI}$ & HR & $95 \% \mathrm{CI}$ & HR & $95 \% \mathrm{CI}$ \\
\hline \multirow{4}{*}{ Meal frequency } & 3 times/day & 1558 & 71.6 & 14 & 60.9 & 14,767 & 1.00 & & 1.00 & & 1.00 & \\
\hline & 2 times/day & 394 & 18.1 & 5 & 21.7 & 3668 & 1.51 & $0.54-4.24$ & 1.71 & $0.60-4.81$ & 1.65 & $0.57-4.76$ \\
\hline & Irregular ${ }^{a}$ & 208 & 9.6 & 4 & 17.4 & 1992 & 2.22 & $0.73-6.78$ & 1.95 & $0.64-5.98$ & 1.73 & $0.53-5.70$ \\
\hline & $p$ for trend & & & & & & 0.15 & & 0.18 & & 0.28 & \\
\hline \multirow{4}{*}{ Breakfast frequency } & Always & 1428 & 65.6 & 10 & 43.5 & 13,511 & 1.00 & & 1.00 & & 1.00 & \\
\hline & Often & 554 & 25.5 & 11 & 47.8 & 5257 & 3.05 & $1.28-7.25$ & 2.86 & $1.20-6.79$ & 2.98 & $1.21-7.33$ \\
\hline & Not at all & 186 & 8.5 & 2 & 8.7 & 1732 & 1.75 & $0.38-8.14$ & 1.58 & $0.34-7.35$ & 1.83 & $0.36-9.21$ \\
\hline & $p$ for trend & & & & & & 0.07 & & 0.10 & & 0.07 & \\
\hline \multirow{4}{*}{ Meal time regularity } & Regular & 967 & 44.4 & 12 & 52.2 & 9118 & 1.00 & & 1.00 & & 1.00 & \\
\hline & Often & 1043 & 47.9 & 7 & 30.4 & 9932 & 0.56 & $0.22-1.44$ & 0.62 & $0.24-1.60$ & 0.60 & $0.23-1.58$ \\
\hline & Irregular & 158 & 7.3 & 4 & 17.4 & 1442 & 2.20 & $0.70-6.94$ & 3.10 & $0.96-9.99$ & 2.28 & $0.64-8.10$ \\
\hline & $p$ for trend & & & & & & 0.76 & & 0.49 & & 0.70 & \\
\hline \multirow{4}{*}{ Meal speed } & Slow & 238 & 10.9 & 5 & 21.7 & 2195 & 1.00 & & 1.00 & & 1.00 & \\
\hline & Average & 1116 & 51.3 & 10 & 43.5 & 10,533 & 0.43 & $0.15-1.24$ & 0.52 & $0.18-1.54$ & 0.46 & $0.15-1.42$ \\
\hline & Fast & 820 & 37.7 & 8 & 34.8 & 7827 & 0.46 & $0.15-1.41$ & 0.56 & $0.18-1.72$ & 0.45 & $0.13-1.54$ \\
\hline & $p$ for trend & & & & & & 0.30 & & 0.42 & & 0.30 & \\
\hline \multirow{4}{*}{ Overeating } & Less than once a week & 793 & 36.4 & 6 & 26.1 & 7477 & 1.00 & & 1.00 & & 1.00 & \\
\hline & 2-3 times a week & 1203 & 55.3 & 14 & 60.9 & 11,426 & 1.38 & $0.55-3.46$ & 1.34 & $0.54-3.37$ & 1.23 & $0.47-3.24$ \\
\hline & More than 4 times a week & 174 & 8.0 & 2 & 8.7 & 1618 & 1.40 & $0.29-6.83$ & 1.47 & $0.30-7.14$ & 1.11 & $0.21-5.92$ \\
\hline & $p$ for trend & & & & & & 0.38 & & 0.36 & & 0.52 & \\
\hline \multirow{4}{*}{ Added spices } & Often & 289 & 13.3 & 2 & 8.7 & 2797 & 1.00 & & 1.00 & & 1.00 & \\
\hline & Sometimes & 1182 & 54.3 & 17 & 73.9 & 11,214 & 2.20 & $0.51-9.52$ & 1.93 & $0.44-8.49$ & 2.47 & $0.54-11.28$ \\
\hline & Never & 692 & 31.8 & 4 & 17.4 & 6437 & 0.91 & $0.17-4.95$ & 0.79 & $0.14-4.40$ & 1.14 & $0.20-6.60$ \\
\hline & $p$ for trend & & & & & & 0.50 & & 0.41 & & 0.73 & \\
\hline
\end{tabular}

Abbreviations are as follows: CI, confidence interval; HR, hazard ratio. ${ }^{a}$ Less than one time a day; ${ }^{\mathrm{b}}$ Adjusted for age, education group (elementary school or less, middle school, high school, college or higher), and breast benign tumor history (yes, no); ${ }^{c}$ Adjusted for diverse potential confounders: total: age, BMI $\left(<23.0,23.0\right.$ to $\left.<25.0, \geq 25 \mathrm{~kg} / \mathrm{m}^{2}\right)$, family history of breast

cancer (yes, no), smoking status (current, ex, non-smoker), alcohol consumption (current, ex, non-drinker), physical activity (yes, no), age at menarche $(\leq 13,14,15, \geq 16$ years), parity $(0,1$,

$2, \geq 3$ ), oral contraceptive use (yes, no), benign breast tumor history (yes, no), hormone use (yes, no), and age at menopause $(<46,46-48,49-51, \geq 52$ years). 


\section{Discussion}

This prospective cohort study investigated the impact of dietary factors on the risk of breast cancer. After adjusting for confounding factors, grilled meat intake was proportionally associated with breast cancer risk in the all and postmenopausal groups, high cholesterol food intake was associated with a higher risk of breast cancer in all women, and meal irregularity was positively associated with breast cancer risk in the all and premenopausal groups.

The higher risk of breast cancer associated with grilled meat consumption is speculated to be due to carcinogenic mutagens such as heterocyclic amines (HCAs) and polycyclic aromatic hydrocarbons (PAHs), which are highly abundant in meat cooked at high temperatures, especially that which is grilled or barbecued [36,37]. HCAs are formed when creatinine, amino acids, and sugars present in meat muscles react at high temperatures [38]. PAHs are produced on or near the surface of meat when meat is cooked directly over an open flame and fat is pyrolyzed or when imperfectly combusted carbon and hydrogen from the fat fall onto hot coals and produce smoke [39]. One of the most common causes of PAH exposure in females is grilled food intake [40]. Although a few studies have shown a non-significant association of grilled meat consumption with breast cancer risk in both all women [41] and postmenopausal women [42], a similar trend to the one observed in this study was identified in several other studies. In case-control studies, grilled meat was associated with a higher risk of breast cancer in all women $[43,44]$. In postmenopausal women, a prospective study showed a borderline significantly higher breast cancer risk associated with the consumption of grilled/pan-fried/well-done meat [45], and a case-control study also showed a higher risk for total lifetime intake of grilled/barbecued beef, pork, and lamb [46]. The association of grilled meat with a higher risk of breast cancer in postmenopausal women may be due to differences in estrogen metabolism pathways based on menopausal status [41,47]. Before menopause, the ovaries are the major estrogen sites [48], whereas after menopause, adipose tissue plays a crucial role in synthesizing estrogen [47]. However, the mechanisms of the association between menopausal status and breast cancer and the interactions with diet remain unclear [49]. Fried foods also generate PAHs and HCAs [50], but they were not found to be associated with breast cancer risk in our studies. Previous studies showed inconsistent outcomes. One cohort study indicated that pan-fried meat was not significantly associated with breast cancer risk in postmenopausal women [42], although in other case-control studies, fried meat was associated with a higher risk in all women $[43,44]$.

Other byproducts of grilled meat are advanced glycation end products (AGEs), which are created from a Maillard or browning reaction-non-enzymatic glycosylation of reducing sugars interacting with free amino groups of proteins, lipids, or nucleic acid [51,52]. By cross-linking with body proteins or binding with cell surface receptors, AGEs induce oxidative stress and inflammation and are therefore associated with dietary-related chronic diseases, including cancer [51]. $N^{\epsilon}$-carboxymethyllysine (CML) AGE is a biologically and chemically well-defined marker for analysis [53]. One study analyzed CML levels in 549 foods and concluded that, although AGEs are naturally present in animal foods, high-temperature cooking methods, especially frying, broiling, grilling, and roasting, produced more AGEs [51]. Some studies have used this database for analysis, and higher dietary CML was associated with pancreatic cancer in men [53], Alzheimer's disease [54], and Barrett's esophagus [55]. However, previous studies regarding breast cancer and AGEs have been limited to AGE receptor status [56,57] and the level of AGEs accumulated in breast tumors [58] to predict breast cancer progression rather than dietary AGE associations.

High-cholesterol food intake was associated with a higher breast cancer risk in the all women in the age-adjusted model and in multivariate Model 2, but this association was not found in multivariate Model 1. The role of dietary cholesterol in breast cancer remains unclear [59,60]. However, several in vivo and in vitro experiments have found that cholesterol may function as a signaling molecule in cancer cells as a cholesterol metabolite, 27-hydroxycholesterol (27HC), was found to act as an antagonist blocking estrogen receptor (ER) activation in the cardiovascular system and also functions as an agonist activating ER+ breast cancer [61]. Therefore, when cholesterol is converted to 
27HC, it may induce ER+ breast cancer growth [61]. In several case-control studies, a study primarily consisting of postmenopausal women and two studies of all women showed that excessive cholesterol intake was associated with a higher risk of breast cancer [62-64]. In a cohort study, the baseline premenopausal women who remained premenopausal at the time of the last biennial questionnaire before censoring or the end of the follow-up also revealed that dietary cholesterol was associated with a higher risk of breast cancer [65]. However, in several cohort studies, no associations were found in all women [66-68] or postmenopausal women [69,70]. When the studies on dietary cholesterol and breast cancer risk were pooled in a meta-analysis, a negative association was found with breast cancer risk in all and premenopausal women [71].

In our study, the animal fat-rich food and fast food groups primarily consisted of processed meat products. Nitrate and nitrite, which are abundant in processed meats, are precursors of $N$-nitroso compounds (NOCs), which are potential carcinogens [72]. However, our study found no association between these food groups and breast cancer risk. Several previous studies also showed inconsistent trends. Processed meat intake was found to be associated with a higher risk of breast cancer among all women in case-control [41] and cohort studies [72,73], in postmenopausal women in nested case-control studies [74], and in both all women and in all subgroups of pre- and postmenopausal women in a cohort study [19]. By contrast, no association was observed between processed meat intake and the likelihood of breast cancer in all women in case-control [75] and cohort studies [76], in postmenopausal women in cohort studies [42,77], and in both all and pre- and postmenopausal women in cohort studies [78,79].

Light-colored, green-yellow, and salted vegetables and fruits were included in the category of fruits and vegetables. Although these food groups showed non-significant associations with the risk of breast cancer, several studies have indicated significant associations of foods other than salted vegetables. Vegetable intake may decrease the risk of breast cancer, but no association has been identified for fruits $[22,80,81]$. Similar results were reported in a meta-analysis of Korean studies as light-colored and green-yellow vegetable intake reduced the risk of breast cancer in all women, whereas fruit intake did not [29]. Among postmenopausal women reporting the highest lifetime intake of grilled/barbequed meat, those with the lowest intake of fruit and vegetables were associated with a significantly higher total lifetime risk [46]. However, a Korean case-control study found that the consumption of pickled vegetables was associated with a higher breast cancer risk in all women [81]. Other plant-based foods and soybean and its products were considered healthy protein foods. The intake of soy foods, including total soy products, soybean curd, and soymilk, showed a decreased risk of breast cancer in a Korean meta-analysis [29]. Our study observed no association of seafood, including seaweed and fish in the healthy protein food group and bonefish, with the risk of breast cancer. In a meta-analysis in Korea, a reduced risk of breast cancer was reported for seaweed, but not for fish consumption [29]. However, a case-control study in Korea identified a decreased risk with a combined vegetable-seafood dietary pattern [82].

We found no association between cereal consumption and breast cancer risk. Several cohort studies of postmenopausal women also showed non-significant associations of whole grain [83] and refined grain consumption [83,84], but conflicting results regarding bread and cereal were found in a case-control study [85]. Sweet foods also showed non-significant associations in our study, but other case-control studies reported different results. One study indicated a borderline significantly higher risk of early-stage breast cancer in the group with the highest intake of sweets [86]. Furthermore, another study that classified sources of sweets found that a higher intake of biscuits, sugar, and chocolate as food items and both desserts and sugars as a food group was associated with a higher risk of breast cancer and hypothesized that this was due to reduced diversity in the diet and increased meal frequency [87].

Dairy foods were not associated with breast cancer risk in our study. In a meta-analysis of 12 prospective studies, total dairy food intake was inversely associated with breast cancer, with low-fat dairy intake showing stronger associations in premenopausal women in particular [88]; however, a meta-analysis of Korean studies reported non-significant results [29]. The intake of caffeinated 
drinks also exhibited non-significant associations in our study. In a 22-year follow-up cohort study, no significant association of caffeinated drink intake with breast cancer risk was identified in all women, but a slightly reduced risk was observed in postmenopausal women [89].

Of the six dietary habits assessed, meal irregularity was associated with a higher risk of breast cancer in all and premenopausal women. However, no previous studies have addressed the direct association between regular eating and breast cancer risk. One study identified an interaction between eating frequency and an inflammatory biomarker, C-reactive protein (CRP), as a putative factor associated with breast cancer. This study showed that a higher eating frequency was inversely proportional to serum CRP levels, suggesting that eating more frequently may lower systemic inflammation and subsequently reduce breast cancer risk [90].

Adding spices to food was not associated with breast cancer risk. In a case-control study, adding table salt was a non-significant factor [75]. Psychological interruption may have affected this result. Subjects already recognize that a higher intake of sodium can increase the risk of chronic diseases, including cancer, and those who are concerned about their health may consequently select a lower frequency in the questionnaire. However, although the HR was not significant, the $P$ for trend was significant in all women after adjusting for covariates. This result indicates that adding less spice may decrease the risk of breast cancer.

Our study has certain strengths: it is a cohort study with a follow-up period of more than nine years. We examined dietary factors at baseline when the subjects did not have breast cancer, so the reports were not subject to selection or recall bias. Additionally, as breast cancer is known to have a long latency, it is important to evaluate dietary patterns prior to the development of disease when environmental exposure may play a strong role [91], indicating that a cohort design would be more meaningful. Additionally, to our knowledge, this is the first prospective study conducted in Korea to address the associations between dietary habits and the risk of breast cancer.

Several limitations should also be mentioned: (1) Our FFQ data cover only baseline information and cannot reflect changes in dietary factors after baseline; (2) Additionally, residual confounding variables may have affected our results. Baseline nutritional status and genetic susceptibility may interact with diet and influence its relationship with breast cancer [25]; however, we could not adjust for these factors; (3) The food dataset included only frequency information, not portion size. Therefore, the intake amount could not be calculated, making it difficult to divide the study subjects into similar groups by the amount of intake of each food item; (4) Although the follow-up period was longer than nine years, there was still an insufficient number of breast cancer cases to infer strong statistical power; (5) The FFQ was limited to 16 items, making it difficult to ensure that all items of certain food groups were measured. Food items in a group may have different associations with breast cancer risk (e.g., red meat versus soy). A potential solution for this problem would be dividing the food group into corresponding food sources to specify their precise influences (e.g., rather than evaluating the consumption frequency of the healthy protein group, it is more appropriate to identify the intake frequency of each food item, such as soybean products, lean meat, fish, and egg whites); (6) Stratification of breast cancer by specific characteristics should be considered in further studies, especially hormone (estrogen and progesterone) receptor and human epidermal growth factor receptor 2 (HER-2) status [25].

\section{Conclusions}

In conclusion, this study showed that breast cancer risk was proportionally associated with grilled meat intake in all women and in postmenopausal women, with high-cholesterol food intake in all women, and with irregular eating in all and premenopausal women. Further studies with longer follow-up periods that include information on portion size, hormone receptor status, levels of each carcinogen in grilled meat, and classification of food groups by the source are needed. 
Acknowledgments: This research was supported by the Grant-in-Aid for Cancer Research and Control from the National Cancer Center of Korea (1510040).

Author Contributions: J.K. designed the research; J.L. and J.H.K. conducted the research and analyzed the data; J.L. and S.-Y.J. provided essential materials; J.H.K. wrote the paper; J.K. had primary responsibility for the final content. All authors read and approved the final manuscript.

Conflicts of Interest: The authors declare no conflicts of interest.

\section{Appendix A}

$\begin{array}{ll}\begin{array}{l}\text { Food list for the } 16 \text { food groups in the short-form FFQ: } \\ \text { Food group }\end{array} & \text { Food list } \\ \text { Cereals } & \text { Rice, bread, noodles, potatoes, sweet potatoes } \\ \text { Salted vegetables and seafood } & \text { Kimchi, salted fermented seafood, food boiled in soya with spices } \\ \text { Light-colored vegetables } & \text { Bean sprouts, cucumbers, radishes, onions, bellflower } \\ \text { Green-yellow vegetables } & \text { Carrots, spinach, sesame leaf, lettuce, zucchini, dropwort } \\ \text { Seaweed } & \text { Laver, brown seaweed, tangle } \\ \text { Fruit } & \text { Apples, tangerines, grapes, watermelon, strawberries, peaches, pears, fruit juice } \\ \text { Grilled meat } & \text { Grilled ribs, barbecue } \\ \text { Healthy protein foods } & \text { Lean meat, fish, legumes, soy foods (e.g., soybean curd), egg whites } \\ \text { Dairy foods } & \text { Yogurt, cheese, milk } \\ \text { Bony fish } & \text { Anchovies, dried whitebait } \\ \text { Fried foods } & \text { Deep-fried food, stir-fried food } \\ \text { High-cholesterol foods } & \text { Egg yolks, seafood (eel, shrimp, squid), organ meats (from fish or other animals) } \\ \text { Animal fat-rich foods } & \text { High-fat red meat (beef, pork), processed food (ham, sausage), butter } \\ \text { Sweet foods } & \text { Bread, cookies, chocolate, honey, candy, ice cream } \\ \text { Fast foods } & \text { Pizza, chicken, processed food } \\ \text { Caffeinated drinks } & \text { Coffee, black tea, cocoa, coke } \\ \end{array}$

\section{References}

1. Ferlay, J.; Soerjomataram, I.; Dikshit, R.; Eser, S.; Mathers, C.; Rebelo, M.; Parkin, D.M.; Forman, D.; Bray, F. Cancer incidence and mortality worldwide: Sources, methods and major patterns in GLOBOCAN 2012. Int. J. Cancer 2015, 136, E359-E386.

2. Siegel, R.L.; Miller, K.D.; Jemal, A. Cancer statistics, 2017. CA Cancer J. Clin. 2017, 67, 7-30. [CrossRef]

3. Oh, C.M.; Won, Y.J.; Jung, K.W.; Kong, H.J.; Cho, H.; Lee, J.K.; Lee, D.H.; Lee, K.H. Cancer statistics in Korea: incidence, mortality, survival, and prevalence in 2013. Cancer Res. Treat. 2016, 48, 436-450. [CrossRef]

4. Park, S.K.; Kim, Y.; Kang, D.; Jung, E.J.; Yoo, K.Y. Risk factors and control strategies for the rapidly rising rate of breast cancer in Korea. J. Breast Cancer 2011, 14, 79-87. [CrossRef]

5. Doll, R.; Peto, R. The causes of cancer: Quantitative estimates of avoidable risks of cancer in the United States today. J. Natl. Cancer Inst. 1981, 66, 1192-1308. [CrossRef]

6. Kotepui, M. Diet and risk of breast cancer. Contemp. Oncol. 2016, 20, 13-19.

7. Michels, K.B.; Mohllajee, A.P.; Roset-Bahmanyar, E.; Beehler, G.P.; Moysich, K.B. Diet and breast cancer. Cancer 2007, 109, 2712-2749. [CrossRef]

8. Guo, J.; Wei, W.; Zhan, L. Red and processed meat intake and risk of breast cancer: A meta-analysis of prospective studies. Breast Cancer Res. Treat. 2015, 151, 191-198. [CrossRef] [PubMed]

9. Park, B.; Park, S.; Shin, H.R.; Shin, A.; Yeo, Y.; Choi, J.Y.; Jung, K.W.; Kim, B.G.; Kim, Y.M.; Noh, D.Y. Population attributable risks of modifiable reproductive factors for breast and ovarian cancers in Korea. BMC Cancer 2016, 16, 1-8. [CrossRef] [PubMed]

10. Kim, Z.; Min, S.Y.; Yoon, C.S.; Lee, H.J.; Lee, J.S.; Youn, H.J.; Park, H.K.; Noh, D.Y.; Hur, M.H. The basic facts of Korean breast cancer in 2011: Results of a nationwide survey and breast cancer registry database. J. Breast Cancer 2014, 17, 99-106. [CrossRef]

11. Lee, S.K.; Sobal, J. Socio-economic, dietary, activity, nutrition and body weight transitions in South Korea. Public Health Nutr. 2003, 6, 665-674. [CrossRef] [PubMed]

12. Paik, N.S. Current status of breast cancer in Korea. Ewha Med. J. 2014, 37, 69-74. [CrossRef]

13. Kim, S.; Moon, S.; Popkin, B.M. The nutrition transition in South Korea. Am. J. Clin. Nutr. 2000, 71, 44-53. [PubMed] 
14. Lee, M.J.; Popkin, B.M.; Kim, S. The unique aspects of the nutrition transition in South Korea: The retention of healthful elements in their traditional diet. Public Health Nutr. 2002, 5, 197-203. [CrossRef] [PubMed]

15. Jun, S.; Ha, K.; Chung, S.; Joung, H. Meat and milk intake in the rice-based Korean diet: Impact on cancer and metabolic syndrome. Proc. Nutr. Soc. 2016, 75, 374-384. [CrossRef] [PubMed]

16. Woo, H.D.; Kim, J. Nutritional epidemiology of cancer in Korea: Recent accomplishments and future directions. Asian Pac. J. Cancer Prev. 2011, 12, 2377-2383. [PubMed]

17. Park, M.K.; Paik, H.Y.; Lee, Y. Intake Trends of red meat, alcohol, and fruits and vegetables as cancer-related dietary factors from 1998 to 2009. Osong Public Health Res. Perspect. 2016, 7, 180-189. [CrossRef] [PubMed]

18. Chen, W.Y.; Rosner, B.; Hankinson, S.E.; Colditz, G.A.; Willett, W.C. Moderate alcohol consumption during adult life, drinking patterns, and breast cancer risk. JAMA 2011, 306, 1884-1890. [CrossRef] [PubMed]

19. Taylor, E.; Burley, V.; Greenwood, D.; Cade, J. Meat consumption and risk of breast cancer in the UK Women's Cohort Study. Br. J. Cancer 2007, 96, 1139-1146. [CrossRef] [PubMed]

20. Kruk, J.; Marchlewicz, M. Dietary fat and physical activity in relation to breast cancer among Polish women. Asian Pac. J. Cancer Prev. 2013, 14, 2495-2502. [CrossRef] [PubMed]

21. Kallianpur, A.R.; Lee, S.A.; Gao, Y.T.; Lu, W.; Zheng, Y.; Ruan, Z.X.; Dai, Q.; Gu, K.; Shu, X.O.; Zheng, W. Dietary animal-derived iron and fat intake and breast cancer risk in the Shanghai Breast Cancer Study. Breast Cancer Res. Treat. 2008, 107, 123-132. [CrossRef] [PubMed]

22. Masala, G.; Assedi, M.; Bendinelli, B.; Ermini, I.; Sieri, S.; Grioni, S.; Sacerdote, C.; Ricceri, F.; Panico, S.; Mattiello, A.; et al. Fruit and vegetables consumption and breast cancer risk: The EPIC Italy study. Breast Cancer Res. Treat. 2012, 132, 1127-1136. [CrossRef] [PubMed]

23. Ferrari, P.; Rinaldi, S.; Jenab, M.; Lukanova, A.; Olsen, A.; Tjønneland, A.; Overvad, K.; Clavel-Chapelon, F.; Fagherazzi, G.; Touillaud, M.; et al. Dietary fiber intake and risk of hormonal receptor-defined breast cancer in the European Prospective Investigation into cancer and nutrition study. Am. J. Clin. Nutr. 2013, 97, 344-353. [CrossRef] [PubMed]

24. Brennan, S.F.; Cantwell, M.M.; Cardwell, C.R.; Velentzis, L.S.; Woodside, J.V. Dietary patterns and breast cancer risk: A systematic review and meta-analysis. Am. J. Clin. Nutr. 2010, 91, 1294-1302. [CrossRef] [PubMed]

25. Romieu, I. Diet and breast cancer. Salud Publica Mexico 2011, 53, 430-439.

26. WCRF/AICR. Food, Nutrition, Physical Activity, and the Prevention of Cancer: A Global Perspective; AICR: Washington, DC, USA, 2007.

27. Hu, F.B. Dietary pattern analysis: A new direction in nutritional epidemiology. Curr. Opin. Lipidol. 2002, 13, 3-9. [CrossRef] [PubMed]

28. Wie, G.A.; Cho, Y.A.; Kang, H.H.; Ryu, K.A.; Yoo, M.K.; Kim, Y.A.; Jung, K.W.; Kim, J.; Lee, J.H.; Joung, H.J. Red meat consumption is associated with an increased overall cancer risk: A prospective cohort study in Korea. Br. J. Nutr. 2014, 112, 238-247. [CrossRef] [PubMed]

29. Woo, H.D.; Park, S.; Oh, K.; Kim, H.J.; Shin, H.R.; Moon, H.K.; Kim, J. Diet and cancer risk in the Korean population: A meta-analysis. Asian Pac. J. Cancer Prev. 2014, 15, 8509-8519. [CrossRef] [PubMed]

30. Shin, A.; Lim, S.Y.; Sung, J.; Shin, H.R.; Kim, J. Dietary intake, eating habits, and metabolic syndrome in Korean men. J. Am. Diet. Assoc. 2009, 109, 633-640. [CrossRef] [PubMed]

31. Shin, A.; Lim, S.; Sung, J.; Myung, S.; Kim, J. Dietary habit and bone mineral density in Korean postmenopausal women. Osteoporos. Int. 2010, 21, 947-955. [CrossRef] [PubMed]

32. Cho, Y.A.; Kim, J.; Cho, E.R.; Shin, A. Dietary patterns and the prevalence of metabolic syndrome in Korean women. Nutr. Metab. Cardiovasc. Dis. 2011, 21, 893-900. [CrossRef] [PubMed]

33. Cho, E.R.; Shin, A.; Lim, S.Y.; Kim, J. Dietary patterns and their associations with health behaviours in Korea. Public Health Nutr. 2011, 14, 356-364. [CrossRef] [PubMed]

34. Farvid, M.S.; Cho, E.; Eliassen, A.H.; Chen, W.Y.; Willett, W.C. Lifetime grain consumption and breast cancer risk. Breast Cancer Res. Treat. 2016, 159, 335-345. [CrossRef] [PubMed]

35. Reeves, G.K.; Pirie, K.; Green, J.; Bull, D.; Beral, V. Reproductive factors and specific histological types of breast cancer: Prospective study and meta-analysis. Br. J. Cancer 2009, 100, 538-544. [CrossRef] [PubMed]

36. Abid, Z.; Cross, A.J.; Sinha, R. Meat, dairy, and cancer. Am. J. Clin. Nutr. 2014, 100, 386S-393S. [CrossRef] [PubMed] 
37. Knize, M.G.; Salmon, C.P.; Pais, P.; Felton, J.S. Food heating and the formation of heterocyclic aromatic amine and polycyclic aromatic hydrocarbon mutagens/carcinogens. Adv. Exp. Med. Biol. 1999, 459, 179-194. [PubMed]

38. Jägerstad, M.; Skog, K. Formation of meat mutagens. Adv. Exp. Med. Biol. 1991, 289, 83-105. [PubMed]

39. Phillips, D.H. Polycyclic aromatic hydrocarbons in the diet. Mutat. Res. Genet. Toxicol. Environ. Mutagen. 1999, 443, 139-147. [CrossRef]

40. White, A.J.; Bradshaw, P.T.; Herring, A.H.; Teitelbaum, S.L.; Beyea, J.; Stellman, S.D.; Steck, S.E.; Mordukhovich, I.; Eng, S.M.; Engel, L.S.; et al. Exposure to multiple sources of polycyclic aromatic hydrocarbons and breast cancer incidence. Environ. Int. 2016, 89, 185-192. [CrossRef] [PubMed]

41. Mourouti, N.; Kontogianni, M.D.; Papavagelis, C.; Plytzanopoulou, P.; Vassilakou, T.; Psaltopoulou, T.; Malamos, N.; Linos, A.; Panagiotakos, D.B. Meat consumption and breast cancer: A case-control study in women. Meat Sci. 2015, 100, 195-201. [CrossRef] [PubMed]

42. Kabat, G.C.; Cross, A.J.; Park, Y.; Schatzkin, A.; Hollenbeck, A.R.; Rohan, T.E.; Sinha, R. Meat intake and meat preparation in relation to risk of postmenopausal breast cancer in the NIH-AARP diet and health study. Int. J. Cancer 2009, 124, 2430-2435. [CrossRef] [PubMed]

43. Fu, Z.; Deming, S.L.; Fair, A.M.; Shrubsole, M.J.; Wujcik, D.M.; Shu, X.O.; Kelley, M.; Zheng, W. Well-done meat intake and meat-derived mutagen exposures in relation to breast cancer risk: The Nashville Breast Health Study. Breast Cancer Res. Treat. 2011, 129, 919-928. [CrossRef] [PubMed]

44. Dai, Q.; Shu, X.O.; Jin, F.; Gao, Y.T.; Ruan, Z.X.; Zheng, W. Consumption of animal foods, cooking methods, and risk of breast cancer. Cancer Epidemiol. Biomark. Prev. 2002, 11, 801-808.

45. Ferrucci, L.; Cross, A.; Graubard, B.; Brinton, L.; McCarty, C.; Ziegler, R.; Ma, X.; Mayne, S.; Sinha, R. Intake of meat, meat mutagens, and iron and the risk of breast cancer in the Prostate, Lung, Colorectal, and Ovarian Cancer Screening Trial. Br. J. Cancer 2009, 101, 178-184. [CrossRef] [PubMed]

46. Steck, S.E.; Gaudet, M.M.; Eng, S.M.; Britton, J.A.; Teitelbaum, S.L.; Neugut, A.I.; Santella, R.M.; Gammon, M.D. Cooked meat and risk of breast cancer-Lifetime versus recent dietary intake. Epidemiology 2007, 18, 373-382. [CrossRef] [PubMed]

47. Siiteri, P.K. Adipose tissue as a source of hormones. Am. J. Clin. Nutr. 1987, 45, 277-282. [PubMed]

48. Clemons, M.; Goss, P. Estrogen and the risk of breast cancer. N. Engl. J. Med. 2001, 344, 276-285. [CrossRef] [PubMed]

49. Cleary, M.P.; Grossmann, M.E. Obesity and breast cancer: The estrogen connection. Endocrinology 2009, 150, 2537-2542. [CrossRef] [PubMed]

50. Balogh, Z.; Gray, J.; Gomaa, E.; Booren, A. Formation and inhibition of heterocyclic aromatic amines in fried ground beef patties. Food Chem. Toxicol. 2000, 38, 395-401. [CrossRef]

51. Uribarri, J.; Woodruff, S.; Goodman, S.; Cai, W.; Chen, X.; Pyzik, R.; Yong, A.; Striker, G.E.; Vlassara, H. Advanced glycation end products in foods and a practical guide to their reduction in the diet. J. Am. Diet. Assoc. 2010, 110, 911-916. [CrossRef] [PubMed]

52. Turner, D. Chapter One-The role of advanced glycation end-products in cancer disparity. Adv. Cancer Res. 2017, 133, 1-22. [PubMed]

53. Jiao, L.; Stolzenberg-Solomon, R.; Zimmerman, T.P.; Duan, Z.; Chen, L.; Kahle, L.; Risch, A.; Subar, A.F.; Cross, A.J.; Hollenbeck, A.; et al. Dietary consumption of advanced glycation end products and pancreatic cancer in the prospective NIH-AARP Diet and Health Study. Am. J. Clin. Nutr. 2015, 101, 126-134. [CrossRef] [PubMed]

54. Perrone, L.; Grant, W.B. Observational and ecological studies of dietary advanced glycation end products in national diets and Alzheimer's disease incidence and prevalence. J. Alzheimers Dis. 2015, 45, 965-979. [PubMed]

55. Jiao, L.; Kramer, J.R.; Chen, L.; Rugge, M.; Parente, P.; Verstovsek, G.; Alsarraj, A.; El-Serag, H.B. Dietary consumption of meat, fat, animal products and advanced glycation end-products and the risk of Barrett's oesophagus. Aliment. Pharmacol. Ther. 2013, 38, 817-824. [CrossRef] [PubMed]

56. Tesarova, P.; Zima, T.; Kubena, A.A.; Kalousova, M. Polymorphisms of the receptor for advanced glycation end products and glyoxalase I and long-term outcome in patients with breast cancer. Tumour Biol. 2017, 39. [CrossRef] [PubMed] 
57. Nankali, M.; Karimi, J.; Goodarzi, M.T.; Saidijam, M.; Khodadadi, I.; Razavi, A.N.E.; Rahimi, F. Increased expression of the receptor for advanced glycation end-products (RAGE) is associated with advanced breast cancer stage. Oncol. Res. Treat. 2016, 39, 622-628. [CrossRef] [PubMed]

58. Nass, N.; Ignatov, A.; Andreas, L.; Weißenborn, C.; Kalinski, T.; Sel, S. Accumulation of the advanced glycation end product carboxymethyl lysine in breast cancer is positively associated with estrogen receptor expression and unfavorable prognosis in estrogen receptor-negative cases. Histochem. Cell Biol. 2017, 147, 625-634. [CrossRef] [PubMed]

59. Danilo, C.; Frank, P.G. Cholesterol and breast cancer development. Curr. Opin. Pharmacol. 2012, 12, 677-682. [CrossRef] [PubMed]

60. Ronco, A.L.; De Stéfani, E.; Stoll, M. Hormonal and metabolic modulation through nutrition: Towards a primary prevention of breast cancer. Breast 2010, 19, 322-332. [CrossRef] [PubMed]

61. Nelson, E.R.; Chang, C.-Y.; McDonnell, D.P. Cholesterol and breast cancer pathophysiology. Trends Endocrinol. Metab. 2014, 25, 649-655. [CrossRef] [PubMed]

62. De Stefani, E.; Deneo Pellegrini, H.; Mendilaharsu, M.; Ronco, A. Essential fatty acids and breast cancer: A case-control study in Uruguay. Int. J. Cancer 1998, 76, 491-494. [CrossRef]

63. Hu, J.; La Vecchia, C.; de Groh, M.; Negri, E.; Morrison, H.; Mery, L. Dietary cholesterol intake and cancer. Ann. Oncol. 2012, 23, 491-500. [CrossRef] [PubMed]

64. Al Othaimeen, A.; Ezzat, A.; Mohamed, G.; Muammar, T.; Al Madouj, A. Dietary fat and breast cancer in Saudi Arabia: A case-control study. East Mediterr. Health J. 2004, 10, 879-886.

65. Farvid, M.S.; Cho, E.; Chen, W.Y.; Eliassen, A.H.; Willett, W.C. Premenopausal dietary fat in relation to pre-and post-menopausal breast cancer. Breast Cancer Res. Treat. 2014, 145, 255-265. [CrossRef] [PubMed]

66. Boeke, C.E.; Eliassen, A.H.; Chen, W.Y.; Cho, E.; Holmes, M.D.; Rosner, B.; Willett, W.C.; Tamimi, R.M. Dietary fat intake in relation to lethal breast cancer in two large prospective cohort studies. Breast Cancer Res. Treat. 2014, 146, 383-392. [CrossRef] [PubMed]

67. Jones, D.Y.; Schatzkin, A.; Green, S.B.; Block, G.; Brinton, L.A.; Ziegler, R.G.; Hoover, R.; Taylor, P.R. Dietary fat and breast cancer in the National Health and Nutrition Examination Survey I epidemiologic follow-up study. J. Natl. Cancer Inst. 1987, 79, 465-471. [CrossRef]

68. Knekt, P.; Albanes, D.; Seppänen, R.; Aromaa, A.; Järvinen, R.; Hyvönen, L.; Teppo, L.; Pukkala, E. Dietary fat and risk of breast cancer. Am. J. Clin. Nutr. 1990, 52, 903-908. [PubMed]

69. Kushi, L.H.; Sellers, T.A.; Potter, J.D.; Nelson, C.L.; Munger, R.G.; Kaye, S.A.; Folsom, A.R. Dietary fat and postmenopausal breast cancer. J. Natl. Cancer Inst. 1992, 84, 1092-1099. [CrossRef] [PubMed]

70. Van den Brandt, P.A.; van't Veer, P.; Goldbohm, R.A.; Dorant, E.; Volovics, A.; Hermus, R.J.; Sturmans, F. A prospective cohort study on dietary fat and the risk of postmenopausal breast cancer. Cancer Res. 1993, 53, 75-82. [PubMed]

71. Li, C.; Yang, L.; Zhang, D.; Jiang, W. Systematic review and meta-analysis suggest that dietary cholesterol intake increases risk of breast cancer. Nutr. Res. 2016, 36, 627-635. [CrossRef] [PubMed]

72. Inoue Choi, M.; Sinha, R.; Gierach, G.L.; Ward, M.H. Red and processed meat, nitrite, and heme iron intakes and postmenopausal breast cancer risk in the NIH-AARP diet and health study. Int. J. Cancer 2016, 138, 1609-1618. [CrossRef] [PubMed]

73. Pala, V.; Krogh, V.; Berrino, F.; Sieri, S.; Grioni, S.; Tjønneland, A.; Olsen, A.; Jakobsen, M.U.; Overvad, K.; Clavel-Chapelon, F.; et al. Meat, eggs, dairy products, and risk of breast cancer in the European Prospective Investigation into Cancer and Nutrition (EPIC) cohort. Am. J. Clin. Nutr. 2009, 90, 602-612. [CrossRef] [PubMed]

74. Egeberg, R.; Olsen, A.; Autrup, H.; Christensen, J.; Stripp, C.; Tetens, I.; Overvad, K.; Tjønneland, A. Meat consumption, $\mathrm{N}$-acetyl transferase 1 and 2 polymorphism and risk of breast cancer in Danish postmenopausal women. Eur. J. Cancer Prev. 2008, 17, 39-47. [CrossRef] [PubMed]

75. Hu, J.; La Vecchia, C.; Morrison, H.; Negri, E.; Mery, L. Salt, processed meat and the risk of cancer. Eur. J. Cancer Prev. 2011, 20, 132-139. [CrossRef] [PubMed]

76. Cross, A.J.; Leitzmann, M.F.; Gail, M.H.; Hollenbeck, A.R.; Schatzkin, A.; Sinha, R. A prospective study of red and processed meat intake in relation to cancer risk. PLoS Med. 2007, 4, e325. [CrossRef] [PubMed]

77. Wu, K.; Sinha, R.; Holmes, M.D.; Giovannucci, E.; Willett, W.; Cho, E. Meat mutagens and breast cancer in postmenopausal women-A cohort analysis. Cancer Epidemiol. Biomark. Prev. 2010, 19, 1301-1310. [CrossRef] [PubMed] 
78. Genkinger, J.M.; Makambi, K.H.; Palmer, J.R.; Rosenberg, L.; Adams-Campbell, L.L. Consumption of dairy and meat in relation to breast cancer risk in the Black Women's Health Study. Cancer Causes Control 2013, 24, 675-684. [CrossRef] [PubMed]

79. Holmes, M.D.; Colditz, G.A.; Hunter, D.J.; Hankinson, S.E.; Rosner, B.; Speizer, F.E.; Willett, W.C. Meat, fish and egg intake and risk of breast cancer. Int. J. Cancer 2003, 104, 221-227. [CrossRef] [PubMed]

80. Gandini, S.; Merzenich, H.; Robertson, C.; Boyle, P. Meta-analysis of studies on breast cancer risk and diet: The role of fruit and vegetable consumption and the intake of associated micronutrients. Eur. J. Cancer 2000, 36, 636-646. [CrossRef]

81. Yu, H.; Hwang, J.Y.; Ro, J.; Kim, J.; Chang, N. Vegetables, but not pickled vegetables, are negatively associated with the risk of breast cancer. Nutr. Cancer 2010, 62, 443-453. [CrossRef] [PubMed]

82. Cho, Y.A.; Kim, J.; Shin, A.; Park, K.-S.; Ro, J. Dietary patterns and breast cancer risk in Korean women. Nutr. Cancer 2010, 62, 1161-1169. [CrossRef] [PubMed]

83. Nicodemus, K.K.; Jacobs, D.R.; Folsom, A.R. Whole and refined grain intake and risk of incident postmenopausal breast cancer (United States). Cancer Causes Control 2001, 12, 917-925. [CrossRef] [PubMed]

84. Egeberg, R.; Olsen, A.; Loft, S.; Christensen, J.; Johnsen, N.F.; Overvad, K.; Tjønneland, A. Intake of whole grain products and risk of breast cancer by hormone receptor status and histology among postmenopausal women. Int. J. Cancer 2009, 124, 745-750. [CrossRef] [PubMed]

85. Franceschi, S.; La Vecchia, C.; Russo, A.; Negri, E.; Favero, A.; Decarli, A. Low-risk diet for breast cancer in Italy. Cancer Epidemiol. Biomark. Prev. 1997, 6, 875-879.

86. Potischman, N.; Coates, R.J.; Swanson, C.A.; Carroll, R.J.; Daling, J.R.; Brogan, D.R.; Gammon, M.D.; Midthune, D.; Curtin, J.; Brinton, L.A. Increased risk of early-stage breast cancer related to consumption of sweet foods among women less than age 45 in the United States. Cancer Causes Control 2002, 13, 937-946. [CrossRef] [PubMed]

87. Tavani, A.; Giordano, L.; Gallus, S.; Talamini, R.; Franceschi, S.; Giacosa, A.; Montella, M.; La Vecchia, C. Consumption of sweet foods and breast cancer risk in Italy. Annal. Oncol. 2006, 17, 341-345. [CrossRef] [PubMed]

88. Dong, J.Y.; Zhang, L.; He, K.; Qin, L.Q. Dairy consumption and risk of breast cancer: A meta-analysis of prospective cohort studies. Breast Cancer Res. Treat. 2011, 127, 23-31. [CrossRef] [PubMed]

89. Ganmaa, D.; Willett, W.C.; Li, T.Y.; Feskanich, D.; van Dam, R.M.; Lopez-Garcia, E.; Hunter, D.J.; Holmes, M.D. Coffee, tea, caffeine and risk of breast cancer: A 22-year follow-up. Int. J. Cancer 2008, 122, 2071-2076. [CrossRef] [PubMed]

90. Marinac, C.R.; Sears, D.D.; Natarajan, L.; Gallo, L.C.; Breen, C.I.; Patterson, R.E. Frequency and circadian timing of eating may influence biomarkers of inflammation and insulin resistance associated with breast cancer risk. PLoS ONE 2015, 10, e0136240. [CrossRef] [PubMed]

91. Mahoney, M.C.; Bevers, T.; Linos, E.; Willett, W.C. Opportunities and strategies for breast cancer prevention through risk reduction. CA Cancer J. Clin. 2008, 58, 347-371. [CrossRef] [PubMed] 